\title{
Developing Martini Coarse-Grained Nitrogen Gas Model for Lipid Nanobubble Simulations
}

\author{
Fujia Tian ${ }^{1,2, \#}$, Xubo Lin ${ }^{1, \#, *}$
}

1. Institute of Single Cell Engineering, Key Laboratory of Ministry of Education for Biomechanics and Mechanobiology, Beijing Advanced Innovation Center for Biomedical Engineering, School of Biological Science and Medical Engineering, Beihang University, Beijing 100191, China.

2. Current Affiliation: Department of Physics, City University of Hong Kong, Hong Kong 999077, China.

*Correspondence to: linxbseu@buaa.edu.cn (XL).
\# These authors contribute equally to this work.
The authors declare no competing financial interest.

\begin{abstract}
Lipid nanobubbles have shown a great potential to be used for the ultrasound molecular imaging and biocompatible drug and gene delivery carriers, which integrate the advantages of both the biocompatibility of lipids and potent physicochemical properties of nanobubbles. Molecular dynamics (MD) simulation provides a powerful tool to investigate fundamental scientific problems related to lipid nanobubbles. With coarse-grained models, the system can be simulated with longer time scale and larger length scale. However, there are very few coarse-grained gas models for lipid nanobubble simulations. Hence, in this work, we developed a simple coarse-grained nitrogen gas model within Martini force field by adjusting the Lennard-Jones interactions of $N_{2}$ with itself, water, lipids, which well reproduced the density of pure $N_{2}$, the density of $N_{2}$ within nanobubbles, and the partitioning thermodynamics of $N_{2}$ in DPPC bilayers. Further lipid nanobubble selfassembly simulation validated the reliability of our coarse-grained $N_{2}$ parameters. Using threecomponent lipid nanobubbles consisting of 1,2-dipalmitoyl-sn-glycero-3-phosphocholine (DPPC), 1,2-dilinoleoyl-sn-glycero-3-phosphocholine (DUPC), and cholesterol, our coarse-grained MD simulations indicated that single-layer membranes could also have clear phase separation, the degree of which was proportional to the radius $(r)$ of the lipid nanobubble, and reached the maximum when $r \rightarrow \infty$ (the planar lipid bi-monolayer at the gas-water interface). Besides, by comparing the planar lipid bi-monolayer (at gas-water interface) and lipid bilayer systems, we found that the latter had much less obvious phase separation. In short, our coarse-grained MD simulations using systems of lipid nanobubbles, lipid bi-monolayers and lipid bilayers will provide useful insights into the role of membrane curvature and interleaflet coupling in the phase separation of multi-component lipid membranes.
\end{abstract}

Keywords: Coarse-Grained Gas Model; Martini Force Field; Molecular Dynamics Simulations; Lipid Nanobubbles. 


\section{Introduction}

Nanobubbles have many unique physicochemical properties and are reported to have better stability than microbubbles ${ }^{[1-2]}$, which show great potential in a series of biomedical applications including ultrasound molecular imaging ${ }^{[3-4]}$, drug/gene delivery ${ }^{[5-6]}$, water treatment ${ }^{[7-8]}$, sonoimmunotherapy ${ }^{[9]}$, and so on. Amphiphilic molecules such as lipids can self-assemble along the air-water interface of nanobubbles. Similar to roles of pulmonary surfactant ${ }^{[10]}$, these molecules can greatly reduce the surface tension of the interface and increase the stability of nanobubbles. As the most abundant component of cell membrane, lipids play vital roles in maintaining its proper structure and functions. Hence, lipid nanobubbles may have excellent biocompatibility. On the other hand, lipid nanobubbles can fuse with cell membrane under a certain intensity of ultrasound ${ }^{[11]}$. Thus, cell membrane can incorporate lipids from lipid nanobubbles ${ }^{[1]}$ and encapsulated gas molecules can re-distribute into the hydrophobic region of cell membrane ${ }^{[12]}$. The former will change the local membrane components, while the latter can decouple the two membrane leaflets and modify membrane structural properties. These two aspects may both dramatically affect the dynamics and functions of membrane proteins, which is so far largely unexplored. In other words, there is plenty of room in revealing the effects of lipid nanobubbles on structure and dynamics of membrane proteins on molecular level, which may greatly expand current biomedical applications of lipid nanobubbles.

Molecular dynamics (MD) simulations provide a powerful tool to investigate the interactions between biomolecules at atomic/near-atomic resolution ${ }^{[13]}$. Many computational efforts have been made to study the stability and dynamics of lipid nanobubbles as well as their interactions with model cell membrane ${ }^{[11,14-16]}$. Especially, coarse-grained models (e.g. Martini ${ }^{[17-19]}$ ) allow MD simulations with much larger length scale and longer time scale than all-atom models, and thus can better capture the complexity of realistic lipid nanobubbles and cell membrane. However, in the current state-of-art coarse-grained MD simulations, gas molecules are modeled as the vacuum in the isothermal-isochoric (NVT) ensemble ${ }^{[11,20-22]}$. This will greatly hinder precise studies on the interactions between lipid nanobubbles and model cell membrane. For one hand, in NVT ensemble, the lateral dimension is fixed, which makes the description of the large membrane deformation unreasonable. For the other hand, gas molecules can permeate into the hydrophobic region of lipid bilayer $^{[12]}$, the replacement of gas molecules with the vacuum could leave possible potent physical effects of these gas molecules on membrane lipids and proteins unclear. Hence, it will be critical to develop coarse-grained models of gas molecules for more precise description of the molecularlevel interactions between lipid nanobubbles and biological systems (Fig. 1).

In this work, based on previous available results of $N_{2}$ gas from atomistic MD simulations ${ }^{[12,}$ 23] and experiments ${ }^{[24-25]}$ (main reason for the choice of $N_{2}$ gas), we developed MARTINIcompatible coarse-grained model of $N_{2}$ gas (Fig. 1), which can well reproduce the lipid nanobubble self-assembly process and overcome possible artifacts of lipid monolayer (e.g. lipid nanobubble ${ }^{[11]}$, pulmonary surfactant ${ }^{[22]}$, tear film ${ }^{[26]}$ ) simulations due to the vacuum treatment of gas molecules simultaneously. With this model, we performed coarse-grained MD simulations of lipid nanobubbles with three different lipid components, and found that obvious liquid-liquid phase separation could appear. Besides, by comparing with lipid bi-monolayer and lipid bilayer systems, 
our results indicated that membrane curvature and interleaflet coupling could both have a strong impact on the phase separation of lipid membrane.

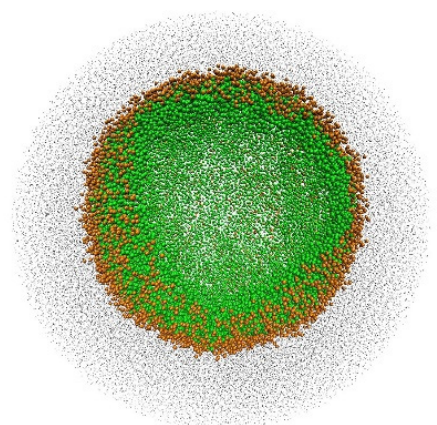

Previous

Lipid Nanobubble MD Simulations (Gas Core was modeled by vacuum)

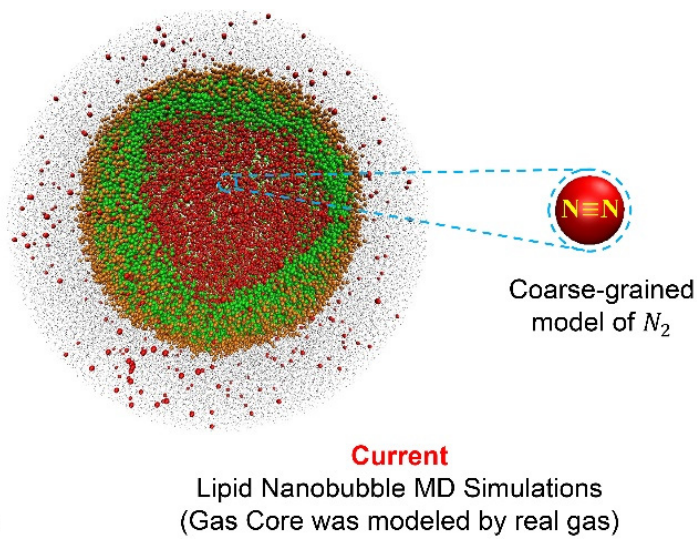

(Gas Core was modeled by real gas)

Figure 1. Coarse-grained gas $\left(N_{2}\right)$ model makes the coarse-grained MD simulations of gas-water interfaces (e.g. lipid nanobubble) more realistic.

\section{Model and Methods}

In this work, coarse-grained parametrization of $N_{2}$ gas within Martini force field is mainly based on reproducing $N_{2}$ gas density $(1.091 \mathrm{~g} / \mathrm{L})^{[24]}$ at body temperature $(\sim 310 \mathrm{~K})$, atomistic simulations of $N_{2}$ nanobubbles ${ }^{[23]}$ and $N_{2}$-lipid membrane interaction systems ${ }^{[12]}$. The obtained coarse-grained $N_{2}$ gas model was further validated in lipid nanobubble self-assembly simulations, which needs to be consistent with the experiments ${ }^{[4,27]}$. Then, coarse-grained MD simulations of lipid nanobubble, lipid bi-monolayer and lipid bilayer systems with the optimized $N_{2}$ gas parameters were performed to reveal effects of membrane curvature and inter-leaflet coupling on the membrane phase separation.

Martini Force Field. As a popular coarse-grained model, Martini force field (version 2.1) ${ }^{[17-18]}$ was used in the current work. In this model, generally 4 heavy atoms are mapped into 1 interaction site (For aromatic compounds, 2 or 3 to 1 mapping rule is applied.), including four main types: polar $(\mathrm{P})$, nonpolar $(\mathrm{N})$, apolar $(\mathrm{C})$, and charged $(\mathrm{Q})$. Four different subtypes $(\mathrm{d}=$ donor, $\mathrm{a}=$ acceptor, $\mathrm{da}=$ both, $0=$ none) are introduced to bead typed of $\mathrm{N}$ and $\mathrm{Q}$ to allow fine representation of the chemical nature. For bead types of $\mathrm{P}$ and $\mathrm{C}$, five different subtypes (from 1, low polarity to 5 , high polarity) are used to describe the degree of polarity. 10 levels of nonbonded interactions using different parameters $(\varepsilon, \sigma)$ for Lennard-Jones (LJ) 12-6 potential energy function $\left(U_{L J}=\right.$ $\left.4 \varepsilon\left[\left(\frac{\sigma}{r}\right)^{12}-\left(\frac{\sigma}{r}\right)^{6}\right]\right)$ are employed to describe the differential nonbonded interactions between different beads (Table S1). In this work, one new coarse-grained bead (G1) was used to represent one $\mathrm{N}_{2}$ gas molecule. For Martini DPPC/DUPC lipids (DPPC: 1,2-dipalmitoyl-sn-glycero-3phosphocholine, DUPC: 1,2-dilinoleoyl-sn-glycero-3-phosphocholine), the head-group consists of two charged beads $(\mathrm{Q} 0,+1 \mathrm{e}$ and $\mathrm{Qa},-1 \mathrm{e})$, the glycerol ester backbone is represented by two nonpolar beads $(\mathrm{Na})$, and each tail contains four apolar beads $(\mathrm{C} 1, \mathrm{C} 1 / \mathrm{C} 4)$. Five different beads (SP1, SC3, SC1, SC1, C1) parameterize cholesterol (Chol) molecules. A polar bead (P4) represents 
one CG water. Systematic parameterization of the nonbonded interactions between G1 and these beads (lipids and water) was performed to reproduce the key physiochemical properties mentioned above.

Molecular Dynamics Simulations. The coarse-grained MD simulations of all systems were performed using GROMACS program v2016.5 ${ }^{[28]}$ and Martini force field ${ }^{[17-18]}$, while the visualization of system snapshots was done using $\mathrm{VMD}^{[29]}$. For all simulations, periodic boundary conditions were applied in three dimensions. The v-rescale thermostat ${ }^{[30]}$ with a relaxation time $\tau$ $=1 p s$ were used to maintain a constant temperature of $310 \mathrm{~K}$ and a constant pressure of 1 bar was kept by Parrinello-Rahman pressure coupling ${ }^{[31]}$ (Coupling constant is $5 \mathrm{ps}$ and compressibility is $5 \times 10^{-5} \mathrm{bar}^{-1}$.) in the NPT ensemble. For systems of pure $N_{2}$ gas, $N_{2}$-water and lipid nanobubbles, isotropic pressure coupling method was used. Meanwhile, semi-isotropic pressure coupling was applied to planar lipid bi-monolayer and lipid bilayer systems. A standard $1.2 \mathrm{~nm}$ cutoff was applied for van der Waals interactions, where the LJ potential was shifted to zero smoothly from 0.9 to 1.2 $\mathrm{nm}$ to reduce the cutoff noise. For columbic potential, a $1.2 \mathrm{~nm}$ cutoff was used for short-range electrostatic interactions while shifting to zero from 0 to $1.2 \mathrm{~nm}$ smoothly. The neighbor list for nonbonded interactions was updated every 10 steps with a cut-off of $1.2 \mathrm{~nm}$.

Trajectory Analysis. Normalized Lateral Contacts of Unsaturated lipids. We first obtained the total number of lateral contacts, $N_{L d}$, among unsaturated lipids DUPC in the phase separated lipid nanobubbles, vesicles and monolayer; contact was defined based on a distance cutoff of $0.6 \mathrm{~nm}$ between any two CG beads of the specified lipid type. Then we normalized $N_{L d}$ by $N_{\text {pure }}$, the total number of lateral contacts in a pure bilayer of DUPC obtained from the last $200 \mathrm{~ns}$ trajectory of a $600 \mathrm{~ns}$ run. Hence, $N_{\text {pure }}$ represents the maximum number of contacts of unsaturated lipids in a fluid bilayer at the same temperature. The normalized number of $L_{d}$ lipid contacts $(N=$ $N_{L d} / N_{\text {pure }}$ ) was used as a proxy for quantifying relative domain size.

Cholesterol Preference. Cholesterol preference was determined based on the number of contacts (cutoff $0.6 \mathrm{~nm})$ of cholesterol with saturated $\left(N_{\mathrm{s}}\right)$ and unsaturated lipids $\left(N_{\mathrm{us}}\right)$ as

$$
\chi_{\mathrm{s}}=\frac{N_{\mathrm{s}} / n_{\mathrm{s}}}{N_{\mathrm{s}} / n_{\mathrm{s}}+N_{\mathrm{us}} / n_{\mathrm{us}}}, \chi_{\mathrm{us}}=\frac{N_{\mathrm{us}} / n_{\mathrm{us}}}{N_{\mathrm{s}} / n_{\mathrm{s}}+N_{\mathrm{us}} / n_{\mathrm{us}}}
$$

where $\chi_{\mathrm{s}}$ and $\chi_{\mathrm{us}}$ are the fraction of cholesterol in contact with saturated and unsaturated lipids, and $n_{\mathrm{s}}$ and $n_{\mathrm{us}}$ are the total number of CG beads of saturated and unsaturated lipids, respectively.

\section{Results and Discussion}

Parametrization of the Nitrogen Gas Model in Martini Force Field. In order to determine the Martini-compatible parameters for coarse-grained $N_{2}$ gas molecules, we firstly calibrated selfinteraction parameters between $N_{2}$ coarse-grained beads (G1) by reproducing the experimental $N_{2}$ gas density $(1.091 \mathrm{~g} / \mathrm{L})$ at body temperature ${ }^{[24]}$. According to Cao's work on the parameterization of $N_{2}$ gas molecules within SAFT- $\gamma$ force field ${ }^{[32]}$, the LJ interaction parameters between $N_{2}$ gas molecules are $\sigma=0.36 \mathrm{~nm}$ and $\varepsilon=0.7 \mathrm{~kJ} / \mathrm{mol}$. In order to evaluate the transferability from SAFT- $\gamma$ coarse-grained $N_{2}$ parameters to Martini coarse-grained $\mathrm{N}_{2}$ parameters, the LJ interaction parameters $\sigma \in[0.28,0.44](\mathrm{nm})(\Delta \sigma=0.02 \mathrm{~nm})$ and $\varepsilon \in[0.2,2.6]$ $(\mathrm{kJ} / \mathrm{mol})(\Delta \varepsilon=0.1 \mathrm{~kJ} / \mathrm{mol})$ for nonbonded interactions between $\mathrm{G} 1$ beads are systematically 
benchmarked for the pure $N_{2}$ gas systems containing $1728 N_{2}$ gas (Initial simulation box size: $6 \times 6 \times 6 \mathrm{~nm}^{3}$. The total number of simulation systems is $9 \times 25=225$.). Each simulation system was run for $50 \mathrm{~ns}$ at $\mathrm{T}=310 \mathrm{~K}$, which added up to a total simulation time of $9 \times 25 \times 50 \mathrm{~ns}=11.25 \mu \mathrm{s}$. The density of $N_{2}$ gas molecules was evaluated over the last 20 ns trajectories as shown in Fig. 2a. Generally, stronger LJ interactions will induce larger $N_{2}$ gas density. And the gas density with $\sigma=0.36 \mathrm{~nm}$ and $\varepsilon=0.7 \mathrm{~kJ} / \mathrm{mol}$ is $1.087 \mathrm{~g} / \mathrm{L}$, which is close to and still able to achieve the exact experimental $N_{2}$ gas density (Fig. 2a). Considering the value of energy parameter $\varepsilon$ is especially critical to the nanobubble formation process, while the value of distance parameter $\sigma$ is less sensitive to the assemble states of $N_{2}$ gas in this range, we finally chose $\sigma=0.36 \mathrm{~nm}$ and $\varepsilon \in[0.7$, 1.9] $\mathrm{kJ} / \mathrm{mol}$, which will be further determined in the following nanobubble formation benchmark simulations.
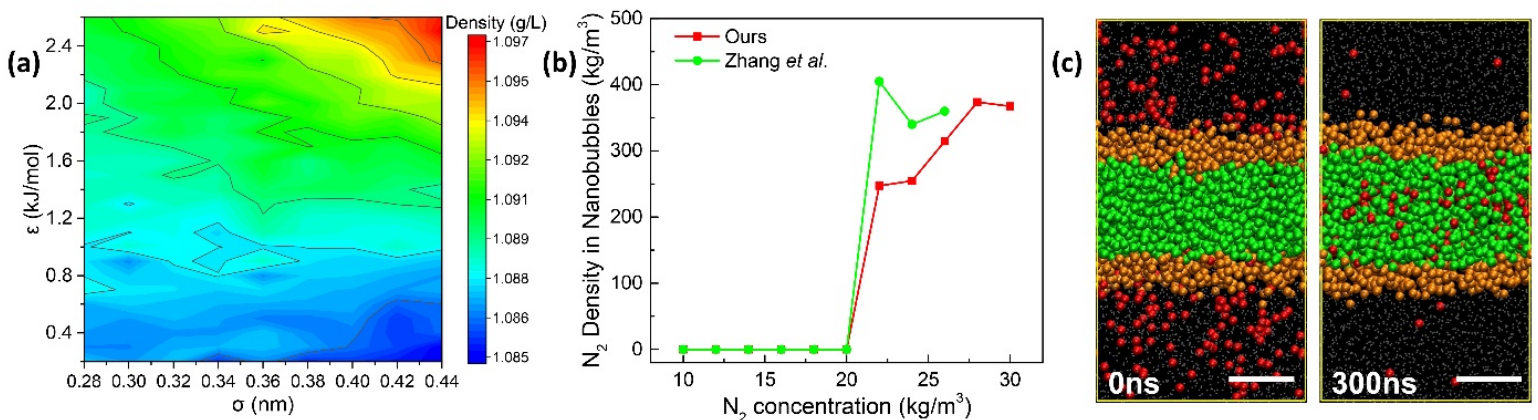

Figure 2. Coarse-grained Parameterization of Nitrogen Gas Molecules: (a) Density of nitrogen molecules calculated from the pure gas simulation systems at $\mathrm{T}=310 \mathrm{~K}$ with different $\sigma(\in[0.28,0.44])$ and $\varepsilon(\in[0.2,2.6])$ values. (b) Optimized parameters ( $\varepsilon$ is 1.6 and $1.4 \mathrm{~kJ} / \mathrm{mol}$ for G1-G1 and G1-P4 LJ interactions respectively) enable better reproduction of the critical concentration for gas nucleation and gas density within the nanobubble in Zhang et al.'s work ${ }^{[23]}$. (c) Optimized parameters ( $\varepsilon$ is 1.4, 1.4, 1.7 and $2.0 \mathrm{~kJ} / \mathrm{mol}$ for G1-Q0, G1-Qa, G1-Na and G1-C1 LJ interactions respectively) well reproduce the preferred localization of $N_{2}$ in DPPC lipid bilayer ${ }^{[12]}$.

In order to obtain the nonbonded interaction parameters between coarse-grained $N_{2}$ beads (G1) and water beads (P4), Lorentz-Berthelot (LB) combination rule $\left(\sigma_{i j}=\left(\sigma_{i i}+\sigma_{i j}\right) / 2, \varepsilon_{i j}(T)=\right.$ $\left(1-k_{i j}\right) \sqrt{\varepsilon_{i i}(T) \varepsilon_{j j}(T)} ; k_{i j}$ is the scaling factor) was used. With the standard LB combination rule $\left(k_{i j}=0, \sigma=0.42 \mathrm{~nm}\right.$ and $\varepsilon=1.9 \mathrm{~kJ} / \mathrm{mol}$ for the nonbonded interactions between $\mathrm{G} 1$ and $\mathrm{P} 4$ beads), it is very difficult to reproduce the nanobubble formation process in water from the coarsegrained MD simulations (Fig. S1). However, this can be corrected by introducing a proper scaling factor $k_{i j}$ for $\varepsilon$ (Tables S2, Fig. S1). In other words, modified value of $\varepsilon$ will be needed. Hence, LJ parameters $\sigma=0.36 \mathrm{~nm}, \varepsilon \in[0.7,1.9] \mathrm{kJ} / \mathrm{mol}$ for G1-G1 and $\sigma=0.42 \mathrm{~nm}, \varepsilon \in[1.1,2.0] \mathrm{kJ} / \mathrm{mol}$ for G1-P4 (Table S3) were carefully calibrated to obtain the optimized energy parameters which can well reproduce the critical $N_{2}$ gas concentration $\left(22 \mathrm{~kg} / \mathrm{m}^{3}\right)$ necessary for the nanobubble formation $^{[23]}$. For each LJ parameter set $(13 \times 10=130$ in total), the $50 \mathrm{~ns}$ benchmark simulations (11 different initial gas concentrations from $10 \mathrm{~kg} / \mathrm{m}^{3}$ to $30 \mathrm{~kg} / \mathrm{m}^{3}, \Delta=2 \mathrm{~kg} / \mathrm{m}^{3}$, Table S4) were 
performed. On average, 8 simulation systems of different initial gas concentrations were performed for each parameter set. In other words, the total simulation time for this part is $13 \times 10 \times 8 \times 50 \mathrm{~ns}=52$ $\mu$ s. As shown in Table S3, LJ parameters of $\sigma=0.36 \mathrm{~nm}, \varepsilon=1.6 \mathrm{~kJ} / \mathrm{mol}$ for G1-G1 and $\sigma=0.42 \mathrm{~nm}$, $\varepsilon=1.4 \mathrm{~kJ} / \mathrm{mol}$ for G1-P4 enable the reproduction of the critical $N_{2}$ gas concentration for the nanobubble formation observed in all-atom MD simulations ${ }^{[23]}$. Using this parameter set, we could also obtain comparable the $N_{2}$ gas density within the nanobubble as Zhang et al.'s work ${ }^{[23]}$ (Fig. 2b). It is worth mention that LJ parameters $\sigma=0.36 \mathrm{~nm}, \varepsilon=1.6 \mathrm{~kJ} / \mathrm{mol}$ for G1-G1 can also achieve the value of $N_{2}$ gas density $\left(1.090 \mathrm{~kg} / \mathrm{m}^{3}\right.$, Fig. 2a), which is closer to the experimental $N_{2}$ gas density at body temperature $\left(1.091 \mathrm{~kg} / \mathrm{m}^{3}\right)$. Hence, the interaction parameters for $N_{2}-N_{2}$ (G1-G1) and $\mathrm{N}_{2}$-water (G1-P4) are fixed.

In order to expand the usage of Martini $N_{2}$ coarse-grained model to lipid systems, we further determined the LJ interaction parameters between $N_{2}$ bead (G1) and lipid coarse-grained beads. The benchmark simulations are mainly based on the united-atom MD simulations of $N_{2}$-DPPC lipid bilayer systems ${ }^{[12]}$. In this work, $\mathrm{Li}$ et al. ${ }^{[12]}$ quantitatively described the partitioning thermodynamics of $N_{2}$ gas molecules into the hydrophobic regions of DPPC lipid bilayer. For our benchmark simulation systems, $N_{2}$ gas molecules (G1 bead) were initially evenly distributed in bulk water ( $\mathrm{P} 4$ bead) region of the DPPC (Q0, Qa, Na, C1 beads) bilayer system. According to Martini interaction parameters (Table S1), Q0 and Qa share almost the same $\varepsilon$ as $\mathrm{P} 4$ interacting with other beads. Hence, the $\varepsilon$ values for G1-Q0 and G1-Qa nonbonded interactions are also fixed as $1.4 \mathrm{~kJ} / \mathrm{mol}$. Besides, the corresponding $\sigma$ values for G1-Q0 and G1-Qa are $0.49 \mathrm{~nm}$, while for G1-C1 and G1-Na give $0.42 \mathrm{~nm}$ based on LB combination rule (the effective size of $\sigma=0.47 \mathrm{~nm}$ is assumed except charged and most apolar beads share $\sigma=0.62 \mathrm{~nm}$ ). According to the nonbonded interaction rules for beads with different polarities in Martini coarse-grained model (Table S1), $\varepsilon \in[1.6,2.0] \mathrm{kJ} / \mathrm{mol}$ for G1-C1 and $\varepsilon \in[1.4,2.0] \mathrm{kJ} / \mathrm{mol}$ for G1-Na LJ interactions (Table S5) were finally evaluated in order to reproduce the distribution of $N_{2}$ gas molecules in the DPPC bilayer systems as indicated by united-atom MD simulations ${ }^{[12]}$. The energy parameter $\varepsilon$ of G1-C1 nonbonded interaction should always be larger than that of G1-Na nonbonded interaction. Therefore, a total of 42 different sets of $\varepsilon$ values were tested for G1-C1 and G1-Na interactions (Table S5). For each parameter set, four lipid bilayer systems with $N_{2}$ gas molecules of different numbers dispersed in bulk waters were performed. The simulation time of each benchmark system was $300 \mathrm{~ns}$, with the last $100 \mathrm{~ns}$ used for the analysis of density profiles. Hence, the total simulation time for this part is $42 \times 4 \times 300 \mathrm{~ns}=50.4 \mu \mathrm{s}$. We finally chose $\varepsilon=2.0 \mathrm{~kJ} / \mathrm{mol}$ for G1-C1 and $\varepsilon=1.7 \mathrm{~kJ} / \mathrm{mol}$ for G1-Na, which properly reproduced the preferred localization of $\mathrm{N}_{2}$ gas molecules (Fig. S2) ${ }^{[12]}$. Besides, we further obtained the nonbonded interactions parameters between $N_{2}$ gas molecules (G1 bead) and DUPC, cholesterol (C4, SP1, SC1 and SC3 beads) based on the above benchmark simulations and parameterization rules for nonbonded interactions in Martini model (Table 1).

Table 1. Finalized nonbonded Interaction Parameters of $N_{2}$ Gas Molecules and Main CG Sites in Martini FF.

\begin{tabular}{ccccc}
\hline Cross-term & $\sigma(\mathrm{nm})$ & $\varepsilon(\mathrm{kJ} / \mathrm{mol})$ & Attractive term & Repulsive term \\
\hline
\end{tabular}




\begin{tabular}{lllll}
\hline G1-G1 & 0.36 & 1.6 & $1.3931 \mathrm{E}-02$ & $3.0326 \mathrm{E}-05$ \\
G1-P4 & 0.42 & 1.4 & $3.0739 \mathrm{E}-02$ & $1.6873 \mathrm{E}-04$ \\
G1-Q0 & 0.49 & 1.4 & $7.7511 \mathrm{E}-02$ & $1.0729 \mathrm{E}-03$ \\
G1-Qa & 0.49 & 1.4 & $7.7511 \mathrm{E}-02$ & $1.0729 \mathrm{E}-03$ \\
G1-Na & 0.42 & 1.7 & $3.7325 \mathrm{E}-02$ & $2.0488 \mathrm{E}-04$ \\
G1-C1 & 0.42 & 2.0 & $4.3912 \mathrm{E}-02$ & $2.4104 \mathrm{E}-04$ \\
G1-C4 & 0.42 & 1.9 & $4.1717 \mathrm{E}-02$ & $2.2898 \mathrm{E}-04$ \\
G1-SP1 & 0.40 & 1.7 & $2.7853 \mathrm{E}-02$ & $1.1409 \mathrm{E}-04$ \\
G1-SC1 & 0.40 & 2.0 & $3.2768 \mathrm{E}-02$ & $1.3422 \mathrm{E}-04$ \\
G1-SC3 & 0.40 & 2.0 & $3.2768 \mathrm{E}-02$ & $1.3422 \mathrm{E}-04$ \\
\hline
\end{tabular}
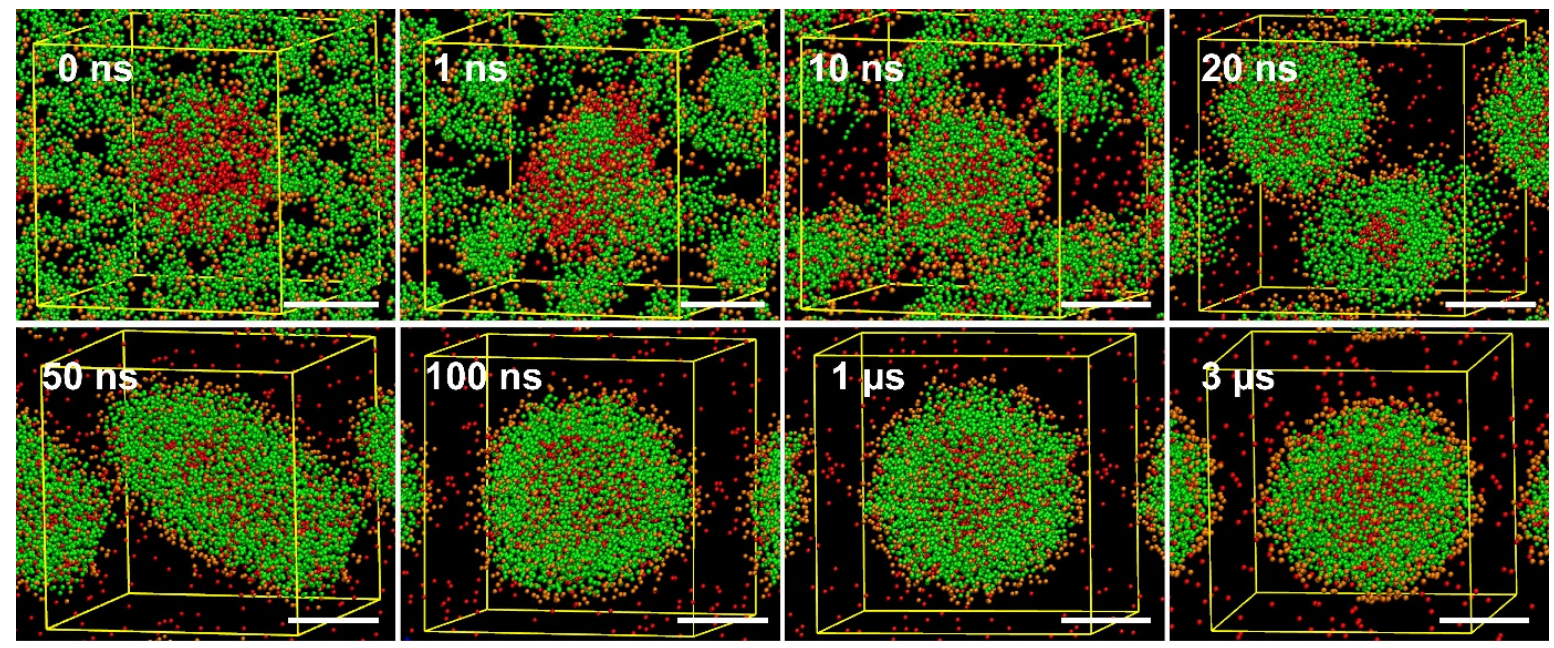

Figure 3. Self-assembly MD Simulations of Lipid Nanobubbles: 256 DPPC molecules were initially randomly distributed around a $N_{2}$ nanobubble (876 molecules), and gradually self-assembled into a singlelayer lipid nanobubble with $N_{2}$ gas core inside and lipid head groups exposed outside. DPPC head groups are colored in orange, DPPC tails in green. Water molecules are not shown for clarity. Scale bar: $3 \mathrm{~nm}$.

\section{Lipid Nanobubble Self-Assembly Simulations Further Validated the Obtained Nitrogen Gas}

Coarse-grained Model. As we know, the lung alveoli provides the key air-water interface for the gas exchange process. The synthetic lipid lamellar bodies and surfactant proteins can be transported to the interface and re-assemble into the lipid monolayer structure ${ }^{[10,22,33]}$. Hence, from the perspective of bionics, the generation of gas bubbles in lipid solution can be one simple experimental method to synthesize lipid micro/nano- bubbles ${ }^{[4,34-35]}$. In other words, the feasibility of the above-obtained $N_{2}$ gas coarse-grained model can be further evaluated by the lipid nanobubble self-assembly simulations. In order for this, we set up the initial simulation systems as follows: (1) $N_{2}$ gas nanobubble, who owns varying radius and number of molecules, was placed at the center of the simulation box $\left(12 \times 12 \times 12 \mathrm{~nm}^{3}\right)$. (2) 180 or 256 DPPC molecules were evenly dispersed in other spaces except the gas nanobubble. (3) The whole system was then solvated by water molecules. In total, 12 self-assembly simulation systems (Table S6) were set up. During the 
pre-equilibrium stage of each simulation, the position of $N_{2}$ gas molecules was constrained, while DPPC and water molecules were fully relaxed. Then, the simulation went through the subsequent $3 \mu$ s production run. For amphiphilic molecules such as DPPC lipids, it is easy to form micelles in water solution $^{[36]}$, which is possible to be converted into spherical vesicles in the absence of gas bubbles $^{[37-38]}$. However, when the hydrophobic $N_{2}$ gas nanobubble existed, dispersed lipids or small lipid clusters could re-assemble along the gas-water interface with hydrophobic lipid tail toward the gas core, and thus form the lipid nanobubble. Fig. 3 shows a typical assembly process for the lipid nanobubble formation in our simulations. First, small lipid clusters and dispersed DPPC molecules were recruited onto the surface of small $N_{2}$ gas nanobubbles within a few ns. Then, lipid-coated $N_{2}$ gas nanobubbles could fuse into an integral lipid nanobubble in tens of ns. Finally, the self-assembled DPPC nanobubble could maintain the stable spherical structure in bulk water for the remaining time of $3 \mu \mathrm{s}$ coarse-grained MD simulations. It is worth mention that insufficient or excessive DPPC molecules can induce lipid nanobubbles of semi-coating or tubular shape correspondingly (Table S6), which is consistent with the principles for self-assembly of lipids ${ }^{[36]}$. In other words, our Martini-compatible coarse-grained model of $N_{2}$ gas molecules could properly reproduce the self-assembly process of the lipid nanobubble formation, which further validated the application feasibility of our $N_{2}$ gas coarse-grained model in lipid-related biological systems. 

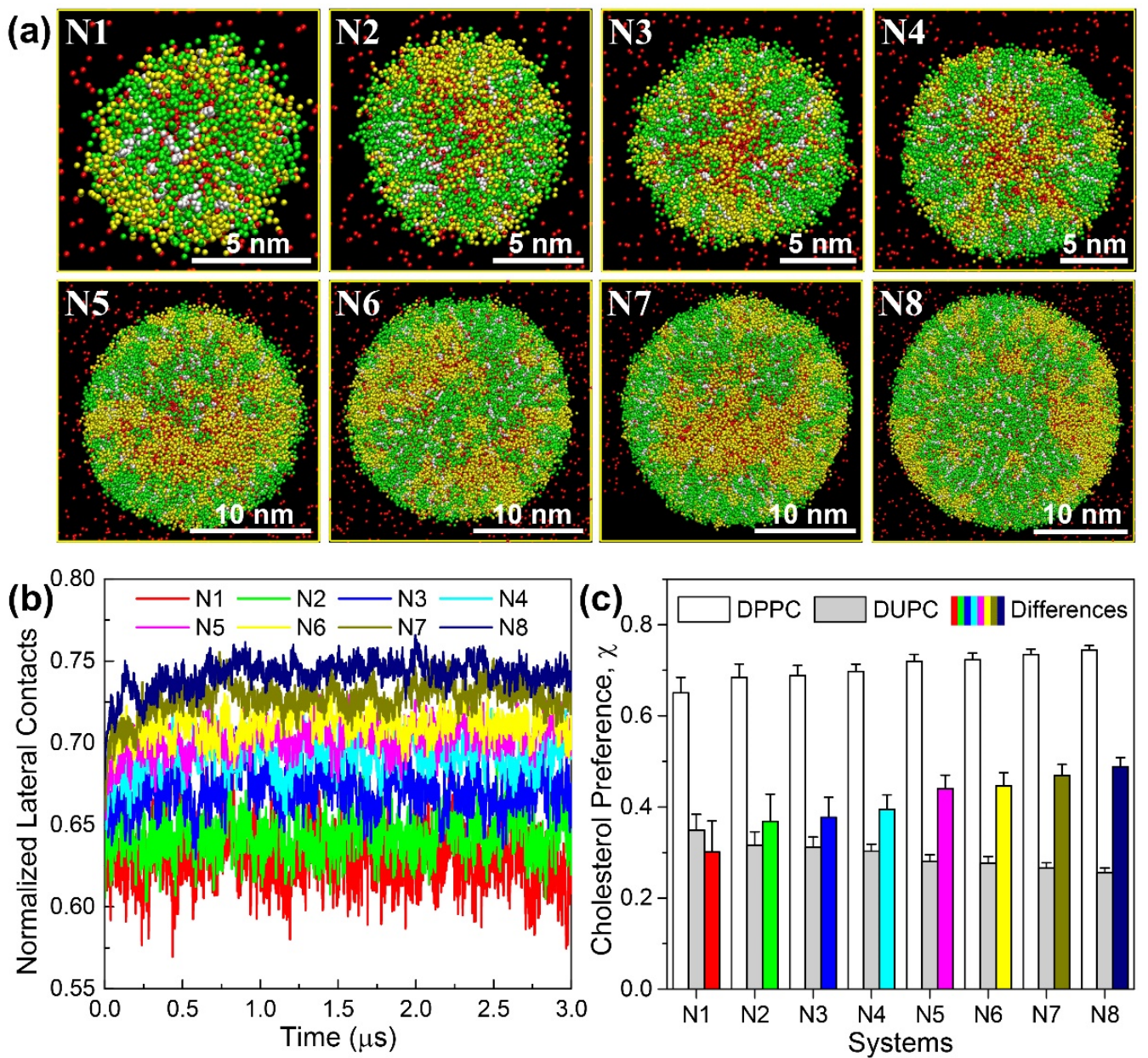

Figure 4. (a) Last frame snapshots of $3 \mu \mathrm{s}$ MD simulations for three-component (DPPC/DUPC/Chol) lipid nanobubbles with increasing inner radius (system N1-N8, inner radius: 3-10 nm, Table S7). DPPC: Green; DUPC: Yellow; Chol: White; Nitrogen: Red. Water is not displayed here. All snapshots are rendered by $\mathrm{VMD}^{[29]}$. (b) Normalized lateral contacts of unsaturated lipids (DUPC) for system N1-N8. (c) Cholesterol preference to different lipids for system N1-N8.

\section{Lipid Nanobubble and Bi-Monolayer Simulations Indicated the Critical Role of Membrane} Curvature in Membrane Phase Separation. The generation of membrane curvature, which can be mediated by lipids, proteins or external stimulus, is necessary in a series of important biological processes for the proper functions of cells such as protein sorting, membrane fusion, organelle shaping and enzyme activation. ${ }^{[39]}$ The presence of local membrane curvature could induce lipid sorting or redistribution ${ }^{[40-42]}$, which would change the local membrane properties to fulfill certain functions. On the other hand, lipid rafts, which are driven by liquid-liquid phase separation of membrane lipids and proteins, also play critical roles in membrane-related biological processes. However, whether and how membrane curvature modulates the size and stability of lipid rafts is still unclear. As discussed above, the spherical lipid nanobubble, which has only one lipid leaflet, may serve as an ideal model system for this purpose. Hence, we set up 8 three-component lipid 
nanobubble systems (system N1-N8) containing DPPC, DUPC and CHOL molecules with the initial inner nanobubble radius ranging from $3 \mathrm{~nm}$ to $10 \mathrm{~nm}$. The system details could be found in Table S7. As shown in Fig. 4a, obvious membrane phase separation appeared in our lipid nanobubbles, which is similar to the cases of lipid vesicles ${ }^{[43-44]}$. Larger lipid nanobubbles (smaller membrane curvature) would have more obvious membrane phase separation, which was further validated by quantifying the normalized lateral contacts of unsaturated lipids (Fig. $4 \mathbf{b}$ ) as well as cholesterol preferences (Fig. 4c). As is known, for the phase separated lipid bilayer system, membrane domains have both the intra-leaflet ${ }^{[43,45-47]}$ and inter-leaflet ${ }^{[48-54]}$ dynamics. Usually, these two kinds of membrane domain dynamics are closely related to each other. In other words, our lipid nanobubbles allow the investigation of effects of membrane curvature on intra-leaflet membrane domain dynamics exclusively.
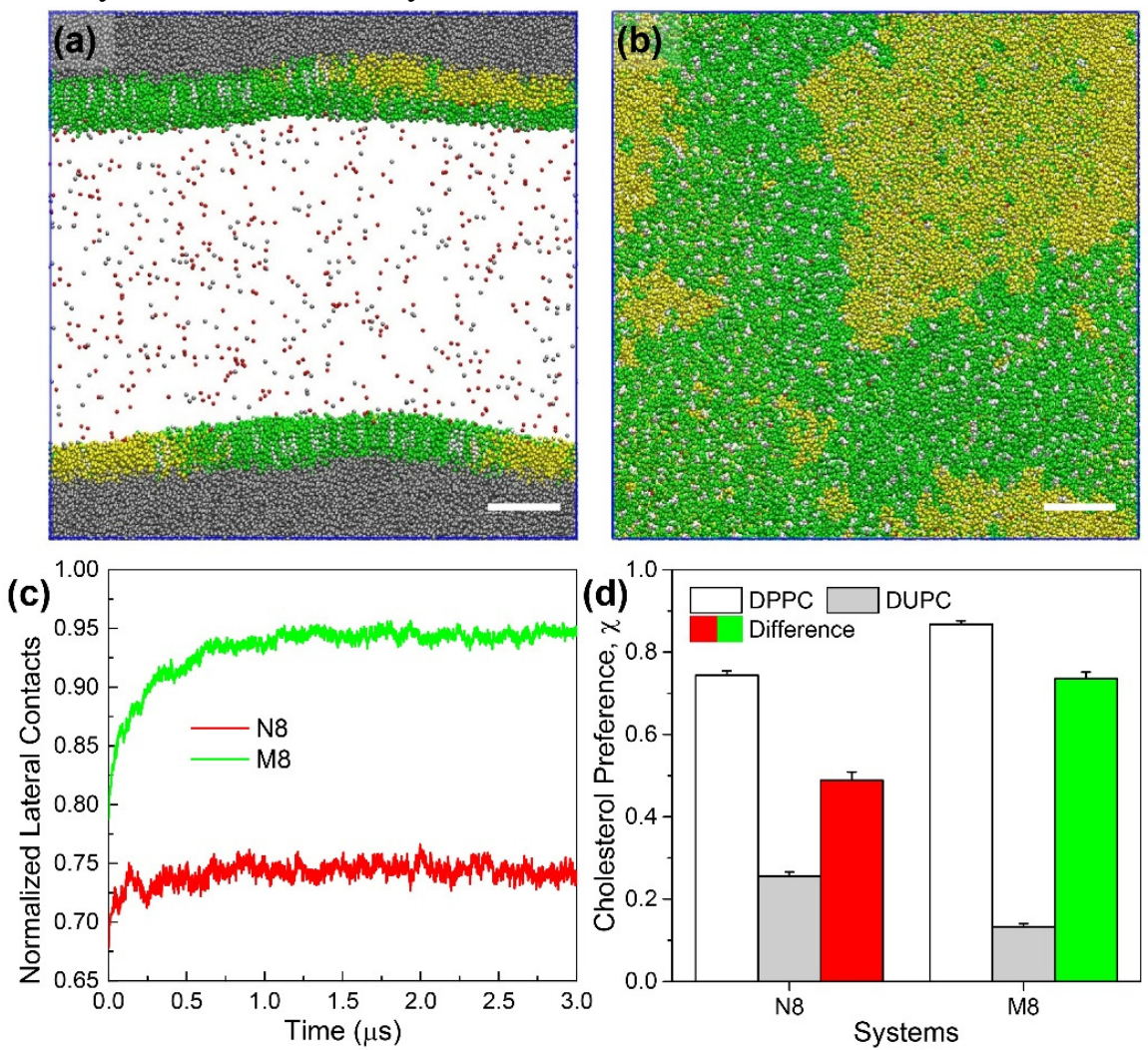

Figure 5. (a) Side-view and (b) top-view system snapshots of the lipid bi-monolayer simulation at $\mathrm{t}=3 \mu \mathrm{s}$ (system M8, Table S8). DPPC is colored in green, DUPC in yellow, CHOL in white, water in gray and $\mathrm{N}_{2}$ in red. (c) Normalized lateral contacts of unsaturated lipids for system N8 and M8. (d) Cholesterol preference to DPPC, DUPC and their differences for system N8 and M8.

As the radius of the lipid nanobubble increases, MD simulations need much more computational resources. Hence, the largest lipid nanobubble we investigated in this work only has the inner radius of $10 \mathrm{~nm}$. In order to make up this shortcoming, we set up the planar lipid bi-monolayer systems (Fig. 5a-b) to simulate the case with the radius $\rightarrow \infty$. System M8 (Table S8) has the same number of lipids and area per lipid in each monolayer as that of system N8. As shown in Fig. 5c-d, the 
results clearly indicated that system M8 had much more obvious membrane phase separation than that of system N8, which further validated the point that the degree of membrane phase separation decreased with the degree of membrane curvature (reciprocal of radius of the curvature). It is worth mention that $N_{2}$ gas molecules were introduced into our lipid bi-monolayer simulations to directly model gas phase, which was widely modeled by the vaccum in previous state-of-art coarse-grained MD simulations of lung surfactant ${ }^{[22,55-58]}$. When the vacuum is used, the z-compressibility of the simulation box has to be zero. With the real gas molecules in the simulations, this setup is no longer necessary. Besides, the explicit interactions between gas molecules and lipids may facilitate the better description of the dynamics of interfacial lung surfactant lipids during the exhalation (monolayer compression) and inhalation (monolayer expansion) processes.
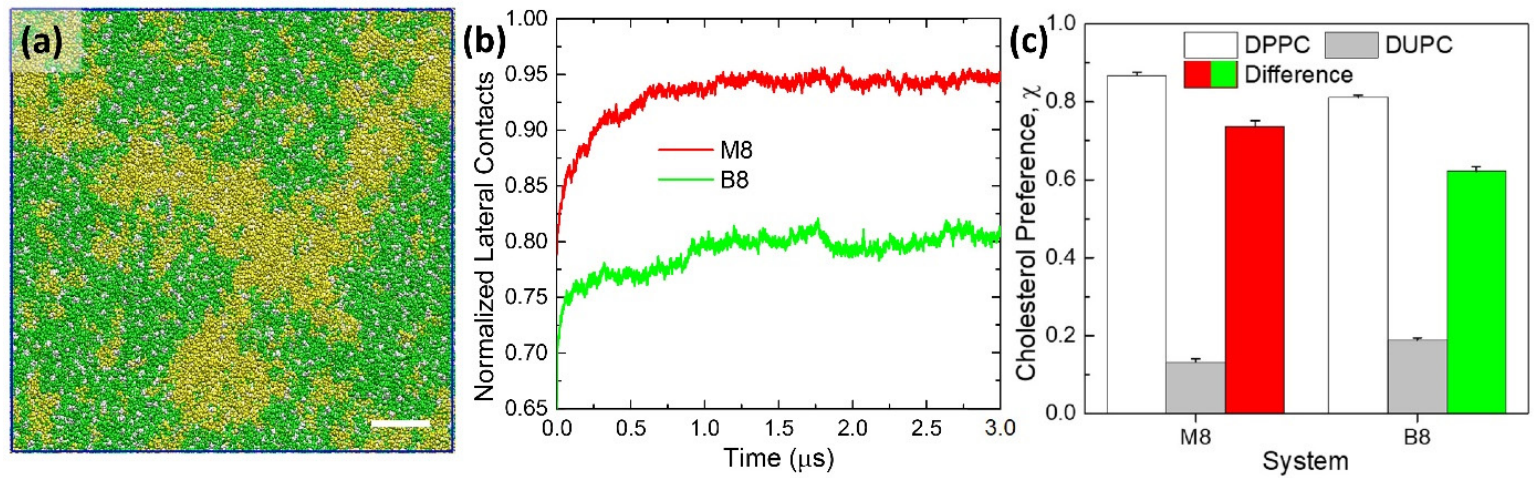

Figure 6. (a) Top-view system snapshot of the lipid bilayer simulation at $\mathrm{t}=3 \mu$ s (system B8, Table S9). DPPC is colored in green, DUPC in yellow, and CHOL in white. (b) Normalized lateral contacts of unsaturated lipids for system B8 and M8. (c) Cholesterol preference to DPPC, DUPC and their differences for system B8 and M8.

\section{Lipid Bi-Monolayer and Bilayer Simulations Demonstrated Inter-leaflet Couplings Modulate} the Membrane Phase Separation. As is discussed above, in the absence of inter-leaflet couplings, obvious membrane phase separation appears in both lipid nanobubble and bi-monolayer systems. Previous studies have indicated that inter-leaflet couplings, which can be regulated by many physicochemical factors ${ }^{[54]}$, modulate membrane domain registration/anti-registration dynamics [48-50]. Whether inter-leaflet couplings can affect the intra-leaflet membrane domain dynamics is still not clear. Here, lipid bi-monolayer and bilayer systems with the same lipid number and comparable area per lipid will allow us to resolve this question directly. As shown in Fig. 6, compared to the lipid bilayer system (system B8, Table S9), lipid bi-monolayer system (system M8, Table S8) has much more obvious membrane phase separation. In other words, the interleaflet couplings may inhibit the intra-leaflet membrane domain dynamics in our simulation systems.

\section{Conclusions}

In this work, based on the density of $N_{2}$ gas molecules ${ }^{[24]}$ as well as previous atomistic MD simulations of $N_{2}$ gas nanobubbles ${ }^{[23]}$ and $N_{2}$-lipid bilayer systems ${ }^{[12]}$, we obtained Martini- 
compatible coarse-grained $N_{2}$ gas model through a series of benchmark simulations, which defined the detailed LJ nonbonded interaction parameters between $N_{2}$ and other molecules (Table 1). The subsequent lipid nanobubble self-assembly simulations further validated the feasibility of our coarse-grained $N_{2}$ gas model. Hence, we applied this model to study the lipid dynamics of three-component lipid nanobubbles. The results indicate that the mixture of saturated and unsaturated lipids as well as cholesterol can go through a clear phase separation in lipid nanobubbles. The larger the lipid nanobubble size, the more obvious the membrane phase separation will be. The lipid bi-monolayer simulations further confirmed the role of membrane curvature in the phase separation of single membrane leaflet. It also showed the feasibility of our gas model for pulmonary surfactant simulations, which may overcome the possible artefacts of using vacuum for the gas phase in previous coarse-grained MD simulations ${ }^{[22,55-58]}$. Besides, by comparing lipid bi-monolayer and bilayer simulations, our results indicated that inter-leaflet coupling could hinder the intra-leaflet membrane domain dynamics. In short, our work shows that the developed coarse-grained $N_{2}$ gas model can be well applied to simulate lipid nanobubbles with complex lipid components and thus related gas-water interface, which are essential for studying the interaction molecular mechanism between lipid nanobubbles and biological systems. However, further efforts are urgently needed to develop coarse-grained models of other common gas molecules for lipid nanobubbles and explore the interactions between lipid nanobubbles (may include proteins and encapsulated drug molecules) and biological systems, which are essential for promoting the wider biomedical applications of lipid nanobubbles.

\section{Acknowledgments}

This work was supported by the National Natural Science Foundation of China (No. 21903002), the Fundamental Research Funds for the Central Universities (No. YWF-20-BJ-J-632), and the Open Fund of State Key Laboratory of Membrane Biology (No. 2020KF09). We are grateful to Center for High Performance Computing of Beihang University (BHHPC) for generous computing resources.

\section{References}

[1] Michailidi, E. D.; Bomis, G.; Varoutoglou, A.; Kyzas, G. Z.; Mitrikas, G.; Mitropoulos, A. C.; Efthimiadou, E. K.; Favvas, E. P. Bulk Nanobubbles: Production and Investigation of Their Formation/Stability Mechanism. J. Colloid Interface Sci. 2020, 564, 371-380.

[2] Nirmalkar, N.; Pacek, A. W.; Barigou, M. On the Existence and Stability of Bulk Nanobubbles. Langmuir 2018, 34, 10964-10973.

[3] Liu, R.; Tang, J.; Xu, Y.; Dai, Z. Bioluminescence Imaging of Inflammation in Vivo Based on Bioluminescence and Fluorescence Resonance Energy Transfer Using Nanobubble Ultrasound Contrast Agent. ACS Nano 2019, 13, 5124-5132.

[4] Fang, K.; Wang, L.; Huang, H.; Lan, M.; Shen, D.; Dong, S.; Guo, Y. Construction of NucleolinTargeted Lipid Nanobubbles and Contrast-Enhanced Ultrasound Molecular Imaging in Triple-Negative Breast Cancer. Pharm. Res. 2020, 37, 145. 
[5] Batchelor, D. V. B.; Abou-Saleh, R. H.; Coletta, P. L.; McLaughlan, J. R.; Peyman, S. A.; Evans, S. D. Nested Nanobubbles for Ultrasound-Triggered Drug Release. ACS Appl. Mater. Interfaces 2020, 12 , 29085-29093.

[6] Zhu, Y.; Zhang, G.; Li, M.; Ma, L.; Huang, J.; Qiu, L. Ultrasound-Augmented Phase Transition Nanobubbles for Targeted Treatment of Paclitaxel-Resistant Cancer. Bioconjugate Chem. 2020, 31, 2008-2020.

[7] Atkinson, A. J.; Apul, O. G.; Schneider, O.; Garcia-Segura, S.; Westerhoff, P. Nanobubble Technologies Offer Opportunities to Improve Water Treatment. Acc. Chem. Res. 2019, 52, 1196-1205.

[8] Agarwal, A.; Ng, W. J.; Liu, Y. Principle and Applications of Microbubble and Nanobubble Technology for Water Treatment. Chemosphere 2011, 84, 1175-1180.

[9] Um, W., et al. Necroptosis-Inducible Polymeric Nanobubbles for Enhanced Cancer Sonoimmunotherapy. Adv. Mater. 2020, 32, 1907953.

[10] Zuo, Y. Y.; Veldhuizen, R. A. W.; Neumann, A. W.; Petersen, N. O.; Possmayer, F. Current Perspectives in Pulmonary Surfactant - Inhibition, Enhancement and Evaluation. Biochim. Biophys. ActaBiomembr. 2008, 1778, 1947-1977.

[11] Sun, D.; Lin, X.; Zhang, Z.; Gu, N. Impact of Shock-Induced Lipid Nanobubble Collapse on a Phospholipid Membrane. J. Phys. Chem. C 2016, 120, 18803-18810.

[12] Li, J.; Zhang, X.; Cao, D. Decoupling of Bilayer Leaflets under Gas Supersaturation: Nitrogen Nanobubbles in a Membrane and Their Implication in Decompression Sickness. J. Phys. D: Appl. Phys. 2018, 51, 184001.

[13] Karplus, M.; McCammon, J. A. Molecular Dynamics Simulations of Biomolecules. Nat. Struct. Biol. 2002, 9, 646-652.

[14] Goliaei, A.; Adhikari, U.; Berkowitz, M. L. Opening of the Blood-Brain Barrier Tight Junction Due to Shock Wave Induced Bubble Collapse: A Molecular Dynamics Simulation Study. ACS Chem. Neurosci. 2015, 6, 1296-1301.

[15] Venable, R. M.; Krämer, A.; Pastor, R. W. Molecular Dynamics Simulations of Membrane Permeability. Chem. Rev. 2019, 119, 5954-5997.

[16] Lu, X.-M.; Yuan, B.; Zhang, X.-R.; Yang, K.; Ma, Y.-Q. Molecular Modeling of Transmembrane Delivery of Paclitaxel by Shock Waves with Nanobubbles. Appl. Phys. Lett. 2017, 110, 023701.

[17] Marrink, S. J.; Risselada, H. J.; Yefimov, S.; Tieleman, D. P.; de Vries, A. H. The Martini Force Field: Coarse Grained Model for Biomolecular Simulations. J. Phys. Chem. B 2007, 111, 7812-7824.

[18] Monticelli, L.; Kandasamy, S. K.; Periole, X.; Larson, R. G.; Tieleman, D. P.; Marrink, S.-J. The Martini Coarse-Grained Force Field: Extension to Proteins. J. Chem. Theory Comput. 2008, 4, 819834.

[19] Marrink, S. J.; Tieleman, D. P. Perspective on the Martini Model. Chem. Soc. Rev. 2013, 42, 68016822.

[20] Min, S. H.; Wijesinghe, S.; Lau, E. Y.; Berkowitz, M. L. Damage to Polystyrene Polymer Film by Shock Wave Induced Bubble Collapse. J. Phys. Chem. B 2020, 124, 7494-7499.

[21] Luo, Z.; Li, S.; Xu, Y.; Yan, Z.; Huang, F.; Yue, T. The Role of Nanoparticle Shape in Translocation across the Pulmonary Surfactant Layer Revealed by Molecular Dynamics Simulations. Environ. Sci.: Nano 2018, 5, 1921-1932. 
[22] Tian, F.; Lin, X.; Valle, R. P.; Zuo, Y. Y.; Gu, N. Poly(Amidoamine) Dendrimer as a Respiratory Nanocarrier: Insights from Experiments and Molecular Dynamics Simulations. Langmuir 2019, 35, 5364-5371.

[23] Zhang, M.; Tu, Y.-s.; Fang, H.-p. Concentration of Nitrogen Molecules Needed by Nitrogen Nanobubbles Existing in Bulk Water. Appl. Math. Mech. -Engl. Ed. 2013, 34, 1433-1438.

[24] Engineering Toolbox. Nitrogen - Density and Specific Weight [Online]. 2018, Available at: https://www.engineeringtoolbox.com/nitrogen-N2-density-specific-weight-temperature-pressured 2039.html [Accessed 15 Dec. 2020].

[25] Zhou, L., et al. Ultrahigh Density of Gas Molecules Confined in Surface Nanobubbles in Ambient Water. J. Am. Chem. Soc. 2020, 142, 5583-5593.

[26] Cwiklik, L. Tear Film Lipid Layer: A Molecular Level View. Biochim. Biophys. Acta-Biomembr. 2016, $1858,2421-2430$.

[27] Krupka, T. M.; Solorio, L.; Wilson, R. E.; Wu, H.; Azar, N.; Exner, A. A. Formulation and Characterization of Echogenic Lipid-Pluronic Nanobubbles. Mol. Pharm. 2010, 7, 49-59.

[28] Abraham, M. J.; Murtola, T.; Schulz, R.; Páll, S.; Smith, J. C.; Hess, B.; Lindahl, E. Gromacs: High Performance Molecular Simulations through Multi-Level Parallelism from Laptops to Supercomputers. Software X 2015, 1-2, 19-25.

[29] Humphrey, W.; Dalke, A.; Schulten, K. Vmd: Visual Molecular Dynamics. J. Mol. Graph. 1996, 14, 33-38.

[30] Bussi, G.; Donadio, D.; Parrinello, M. Canonical Sampling through Velocity Rescaling. J. Chem. Phys. 2007, 126, 014101.

[31] Parrinello, M.; Rahman, A. Polymorphic Transitions in Single Crystals: A New Molecular Dynamics Method. J. Appl. Phys. 1981, 52, 7182-7190.

[32] Cao, F.; Deetz, J. D.; Sun, H. Free Energy-Based Coarse-Grained Force Field for Binary Mixtures of Hydrocarbons, Nitrogen, Oxygen, and Carbon Dioxide. J. Chem. Inf. Model. 2017, 57, 50-59.

[33] Sever, N.; Miličić, G.; Bodnar, N. O.; Wu, X.; Rapoport, T. A. Mechanism of Lamellar Body Formation by Lung Surfactant Protein B. Mol. Cell 2020, doi: 10.1016/j.molcel.2020.10.042.

[34] Tian, J.; Yang, F.; Cui, H.; Zhou, Y.; Ruan, X.; Gu, N. A Novel Approach to Making the Gas-Filled Liposome Real: Based on the Interaction of Lipid with Free Nanobubble within the Solution. ACS Appl. Mater. Interfaces 2015, 7, 26579-84.

[35] Zheng, R.; Yin, T.; Wang, P.; Zheng, R.; Zheng, B.; Cheng, D.; Zhang, X.; Shuai, X.-T. Nanobubbles for Enhanced Ultrasound Imaging of Tumors. Int. J. Nanomed. 2012, 7, 895-904.

[36] Antonietti, M.; Förster, S. Vesicles and Liposomes: A Self-Assembly Principle Beyond Lipids. Adv. Mater. 2003, 15, 1323-1333.

[37] Yang, G.; O'Duill, M.; Gouverneur, V.; Krafft, M. P. Recruitment and Immobilization of a Fluorinated Biomarker across an Interfacial Phospholipid Film Using a Fluorocarbon Gas. Angew. Chem. Int. Ed. Engl. 2015, 54, 8402-8406.

[38] Brea, R. J.; Cole, C. M.; Devaraj, N. K. In Situ Vesicle Formation by Native Chemical Ligation. Angew. Chem. Int. Ed. Engl. 2014, 53, 14102-14105.

[39] McMahon, H. T.; Boucrot, E. Membrane Curvature at a Glance. J. Cell Sci. 2015, 128, 1065-1070.

[40] Beltrán-Heredia, E.; Tsai, F.-C.; Salinas-Almaguer, S.; Cao, F. J.; Bassereau, P.; Monroy, F. Membrane 
Curvature Induces Cardiolipin Sorting. Commun. Biol. 2019, 2, 225.

[41] Lin, X.; Wang, H.; Lou, Z.; Cao, M.; Zhang, Z.; Gu, N. Roles of Pip2 in the Membrane Binding of Mim I-Bar: Insights from Molecular Dynamics Simulations. FEBS Lett. 2018, 592, 2533-2542.

[42] Baoukina, S.; Ingólfsson, H. I.; Marrink, S. J.; Tieleman, D. P. Curvature-Induced Sorting of Lipids in Plasma Membrane Tethers. Adv. Theory Simul. 2018, 1, 1800034.

[43] Risselada, H. J.; Marrink, S. J. The Molecular Face of Lipid Rafts in Model Membranes. Proc. Natl. Acad. Sci. U. S. A. 2008, 105, 17367-17372.

[44] Kaiser, H.-J.; Lingwood, D.; Levental, I.; Sampaio, J. L.; Kalvodova, L.; Rajendran, L.; Simons, K. Order of Lipid Phases in Model and Plasma Membranes. Proc. Natl. Acad. Sci. U. S. A. 2009, 106, 16645-16650.

[45] Lin, X.; Lorent, J. H.; Skinkle, A. D.; Levental, K. R.; Waxham, M. N.; Gorfe, A. A.; Levental, I. Domain Stability in Biomimetic Membranes Driven by Lipid Polyunsaturation. J. Phys. Chem. B 2016, 120, 11930-11941.

[46] Gu, R.-X.; Baoukina, S.; Tieleman, D. P. Phase Separation in Atomistic Simulations of Model Membranes. J. Am. Chem. Soc. 2020, 142, 2844-2856.

[47] Heberle, F. A.; Feigenson, G. W. J. C. S. H. p. i. b. Phase Separation in Lipid Membranes. Cold Spring Harb. Perspect. Biol. 2011, 3, a004630.

[48] Lin, X.; Zhang, S.; Ding, H.; Levental, I.; Gorfe, A. A. The Aliphatic Chain of Cholesterol Modulates Bilayer Interleaflet Coupling and Domain Registration. FEBS Lett. 2016, 590, 3368-3374.

[49] Zhang, S.; Lin, X. Lipid Acyl Chain Cis Double Bond Position Modulates Membrane Domain Registration/Anti-Registration. J. Am. Chem. Soc. 2019, 141, 15884-15890.

[50] Fowler, P. W.; Williamson, J. J.; Sansom, M. S. P.; Olmsted, P. D. Roles of Interleaflet Coupling and Hydrophobic Mismatch in Lipid Membrane Phase-Separation Kinetics. J. Am. Chem. Soc. 2016, 138, 11633-11642.

[51] Seo, S.; Murata, M.; Shinoda, W. Pivotal Role of Interdigitation in Interleaflet Interactions: Implications from Molecular Dynamics Simulations. J. Phys. Chem. Lett. 2020, 11, 5171-5176.

[52] Thallmair, S.; Ingólfsson, H. I.; Marrink, S. J. Cholesterol Flip-Flop Impacts Domain Registration in Plasma Membrane Models. J. Phys. Chem. Lett. 2018, 9, 5527-5533.

[53]Perlmutter, J. D.; Sachs, J. N. Interleaflet Interaction and Asymmetry in Phase Separated Lipid Bilayers: Molecular Dynamics Simulations. J. Am. Chem. Soc. 2011, 133, 6563-6577.

[54] Nickels, J. D.; Smith, J. C.; Cheng, X. Lateral Organization, Bilayer Asymmetry, and Inter-Leaflet Coupling of Biological Membranes. Chem. Phys. Lipids 2015, 192, 87-99.

[55] Hu, G.; Jiao, B.; Shi, X.; Valle, R. P.; Fan, Q.; Zuo, Y. Y. Physicochemical Properties of Nanoparticles Regulate Translocation across Pulmonary Surfactant Monolayer and Formation of Lipoprotein Corona. ACS Nano 2013, 7, 10525-10533.

[56] Bai, X.; Li, M.; Hu, G. Nanoparticle Translocation across the Lung Surfactant Film Regulated by Grafting Polymers. Nanoscale 2020, 12, 3931-3940.

[57] Xu, Y.; Li, S.; Luo, Z.; Ren, H.; Zhang, X.; Huang, F.; Zuo, Y. Y.; Yue, T. Role of Lipid Coating in the Transport of Nanodroplets across the Pulmonary Surfactant Layer Revealed by Molecular Dynamics Simulations. Langmuir 2018, 34, 9054-9063.

[58] Baoukina, S.; Mendez-Villuendas, E.; Tieleman, D. P. Molecular View of Phase Coexistence in Lipid 
Monolayers. J. Am. Chem. Soc. 2012, 134, 17543-17553. 


\title{
Supporting Information for
}

\section{Developing Martini Coarse-Grained Nitrogen Gas Model for Lipid Nanobubble Simulations}

\author{
Fujia Tian ${ }^{1,2, \#}$, Xubo Lin ${ }^{1, \#, *}$
}

1. Institute of Single Cell Engineering, Key Laboratory of Ministry of Education for Biomechanics and Mechanobiology, Beijing Advanced Innovation Center for Biomedical Engineering, School of Biological Science and Medical Engineering, Beihang University, Beijing 100191, China.

2. Current Affiliation: Department of Physics, City University of Hong Kong, Hong Kong 999077, China.

*Correspondence to: linxbseu@buaa.edu.cn (XL).

\# These authors contribute equally to this work.

The authors declare no competing financial interest. 
Table S1. Lennar-Jones (LJ) interaction Matrix in Martini model. Energy parameters $(\varepsilon \mathrm{kJ} / \mathrm{mol})$ summarized from the work of Marrink et al. ${ }^{[1]}$

\begin{tabular}{|c|c|c|c|c|c|c|c|c|c|c|c|c|c|c|c|c|c|c|}
\hline & C1 & C2 & C3 & C4 & C5 & N0 & Na & Nd & Nda & P1 & P2 & P3 & P4 & P5 & Q0 & Qa & Qd & Qda \\
\hline C1 & 3.5 & 3.5 & 3.5 & 3.1 & 3.1 & 2.7 & 2.7 & 2.7 & 2.7 & 2.7 & 2.3 & 2.3 & 2.0 & 2.0 & 2.0 & 2.0 & 2.0 & 2.0 \\
\hline C2 & & 3.5 & 3.5 & 3.1 & 3.1 & 3.1 & 2.7 & 2.7 & 2.7 & 3.1 & 2.7 & 2.7 & 2.3 & 2.3 & 2.0 & 2.0 & 2.0 & 2.0 \\
\hline C3 & & & 3.5 & 3.5 & 3.5 & 3.5 & 2.7 & 2.7 & 2.7 & 3.5 & 3.1 & 3.1 & 2.7 & 2.7 & 2.3 & 2.3 & 2.3 & 2.3 \\
\hline C4 & & & 3.5 & 3.5 & 3.5 & 3.1 & 3.1 & 3.1 & 3.5 & 3.5 & 3.1 & 2.7 & 2.7 & 2.7 & 2.7 & 2.7 & 2.7 \\
\hline C5 & & & & 3.5 & 3.5 & 3.5 & 3.5 & 3.5 & 3.5 & 3.5 & 3.5 & 3.1 & 3.1 & 3.1 & 3.1 & 3.1 & 3.1 \\
\hline N0 & & & & & 3.5 & 3.5 & 3.5 & 3.5 & 4.0 & 4.0 & 3.5 & 3.5 & 3.5 & 3.5 & 3.5 & 3.5 & 3.5 \\
\hline Na & & & & & & 4.0 & 4.5 & 4.5 & 4.5 & 4.5 & 4.5 & 4.0 & 5.0 & 4.0 & 4.0 & 5.0 & 5.0 \\
\hline Nd & & & & & & & 4.0 & 4.5 & 4.5 & 4.5 & 4.5 & 4.0 & 5.0 & 4.0 & 5.0 & 4.0 & 5.0 \\
\hline Nda & & & & & & & & 4.5 & 4.5 & 4.5 & 4.5 & 4.0 & 5.0 & 4.0 & 5.0 & 5.0 & 5.0 \\
\hline P1 & & & & & & & & & & 4.5 & 4.5 & 4.5 & 4.5 & 5.6 & 4.0 & 5.0 & 5.0 & 5.0 \\
\hline P2 & & & & & & & & & & 4.5 & 4.5 & 4.5 & 5.6 & 4.5 & 5.0 & 5.0 & 5.0 \\
\hline P3 & & & & & & & & & & & 5.0 & 5.0 & 5.6 & 5.0 & 5.6 & 5.6 & 5.6 \\
\hline P4 & & & & & & & & & & & & 5.0 & 5.6 & 5.6 & 5.6 & 5.6 & 5.6 \\
\hline P5 & & & & & & & & & & & & & 5.6 & 5.0 & 5.6 & 5.6 & 5.6 \\
\hline Q0 & & & & & & & & & & & & & & & 3.5 & 4.5 & 4.5 & 4.5 \\
\hline Qa & & & & & & & & & & & & & & & 5.0 & 5.6 & 5.6 \\
\hline Qd & & & & & & & & & & & & & & & & 5.0 & 5.6 \\
\hline Qda & & & & & & & & & & & & & & & & & 5.6 \\
\hline
\end{tabular}

$\sigma=0.47 \mathrm{~nm}$ for all interaction groups except $\sigma=0.62 \mathrm{~nm}$ for $\mathrm{C} 1$ bead interacting with charged beads (Q).

Table S2. Scaling factor and corresponding parameters in LJ potential for non-bonded interaction between nitrogen and water beads.

\begin{tabular}{|c|c|c|c|c|c|}
\hline Number & $\mathbf{k}$ & $\boldsymbol{\sigma}(\mathbf{n m})$ & $\boldsymbol{\varepsilon}(\mathbf{k J} / \mathbf{m o l})$ & Attractive term & Repulsive term \\
\hline $\mathbf{1}$ & 0 & 0.42 & 1.9 & $4.1717 \mathrm{E}-02$ & $2.2898 \mathrm{E}-04$ \\
\hline $\mathbf{2}$ & 0.08 & 0.42 & 1.7 & $3.7325 \mathrm{E}-02$ & $2.0488 \mathrm{E}-04$ \\
\hline $\mathbf{3}$ & 0.12 & 0.42 & 1.6 & $3.5130 \mathrm{E}-02$ & $1.9283 \mathrm{E}-04$ \\
\hline $\mathbf{4}$ & 0.2 & 0.42 & 1.5 & $3.2934 \mathrm{E}-02$ & $1.8078 \mathrm{E}-04$ \\
\hline $\mathbf{5}$ & 0.24 & 0.42 & 1.4 & $3.0739 \mathrm{E}-02$ & $1.6873 \mathrm{E}-04$ \\
\hline $\mathbf{6}$ & 0.28 & 0.42 & 1.3 & $2.8543 \mathrm{E}-02$ & $1.5667 \mathrm{E}-04$ \\
\hline
\end{tabular}



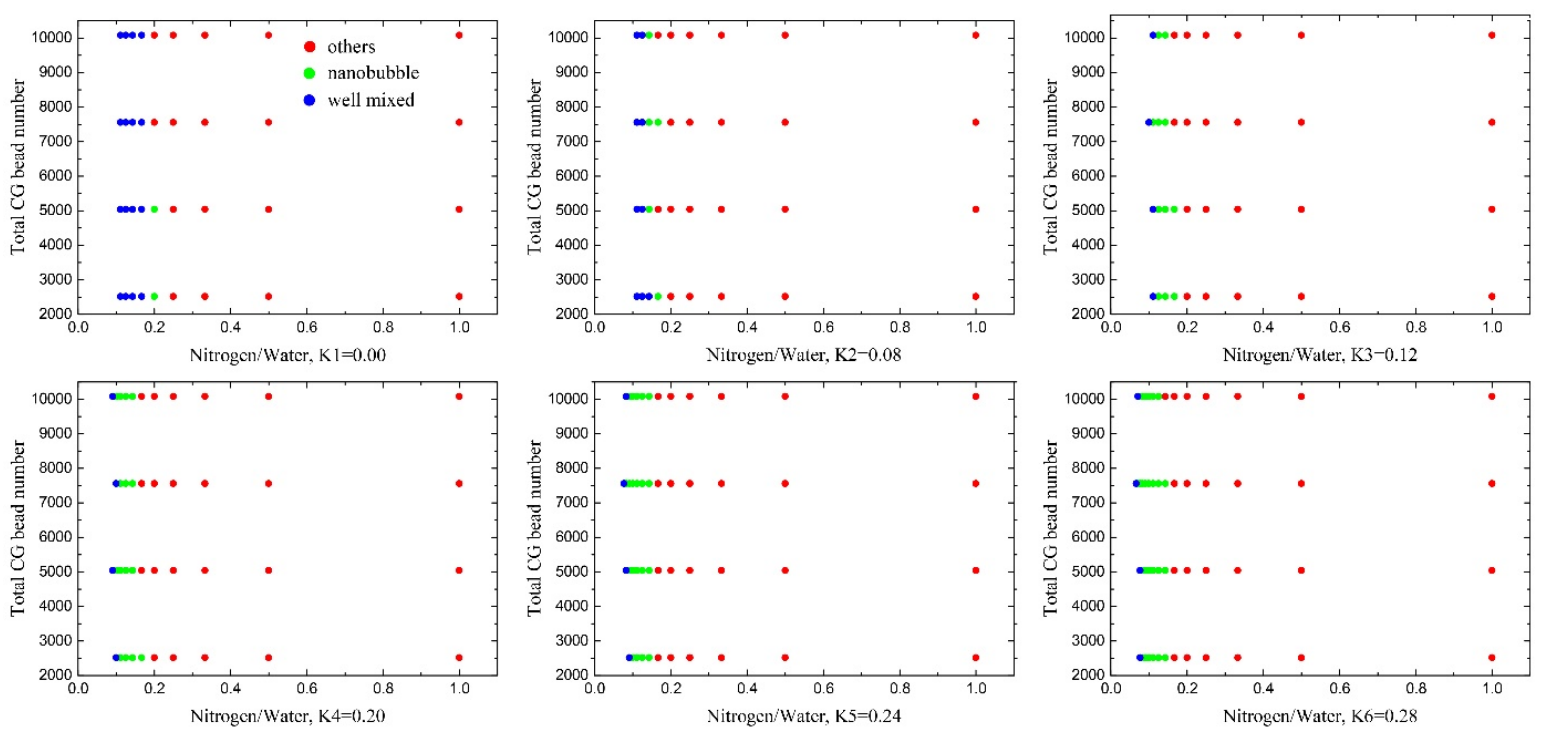

Figure S1. Coarse-grained molecular dynamics (MD) simulations of mixed systems of $N_{2}$ gas and water molecules with different scaling factors. Systems of different size and nitrogen/water ratio were investigated for each scaling factor.

Table S3. Critical concentration $(\mathrm{g} / \mathrm{L})$ for nitrogen nanobubble formation obtained from simulations of nitrogen-water mixtures with different LJ interaction parameter combinations.

\begin{tabular}{|c|c|c|c|c|c|c|c|c|c|c|c|c|c|c|}
\hline & \multicolumn{14}{|c|}{ G1-G1 $(\sigma=0.36 \mathrm{~nm})$} \\
\hline \multirow{11}{*}{$\begin{array}{c}\text { G1-P4 } \\
(\sigma=0.42 \\
\text { nm) }\end{array}$} & $\varepsilon(\mathrm{kJ} / \mathrm{mol})$ & 0.7 & 0.8 & 0.9 & 1.0 & 1.1 & 1.2 & 1.3 & 1.4 & 1.5 & 1.6 & 1.7 & 1.8 & 1.9 \\
\hline & 1.1 & 22 & 22 & 22 & 20 & 20 & 20 & 18 & 18 & 18 & 16 & 14 & 14 & 14 \\
\hline & 1.2 & 24 & 22 & 24 & 24 & 22 & 20 & 20 & 18 & 18 & 16 & 16 & 16 & 14 \\
\hline & 1.3 & 26 & 28 & 26 & 26 & 24 & 24 & 22 & 20 & 20 & 18 & 18 & 16 & 16 \\
\hline & 1.4 & $>30$ & 30 & 30 & 30 & 30 & 28 & 26 & 24 & 24 & 22 & 20 & 20 & 18 \\
\hline & 1.5 & $>30$ & $>30$ & $>30$ & $>30$ & 30 & $>30$ & 30 & 28 & 28 & 26 & 24 & 24 & 20 \\
\hline & 1.6 & $>30$ & $>30$ & $>30$ & $>30$ & $>30$ & $>30$ & $>30$ & $>30$ & $>30$ & 30 & 26 & 26 & 24 \\
\hline & 1.7 & $>30$ & $>30$ & $>30$ & $>30$ & $>30$ & $>30$ & $>30$ & $>30$ & $>30$ & $>30$ & $>30$ & 30 & 26 \\
\hline & 1.8 & $>30$ & $>30$ & $>30$ & $>30$ & $>30$ & $>30$ & $>30$ & $>30$ & $>30$ & $>30$ & $>30$ & $>30$ & $>30$ \\
\hline & 1.9 & $>30$ & $>30$ & $>30$ & $>30$ & $>30$ & $>30$ & $>30$ & $>30$ & $>30$ & $>30$ & $>30$ & $>30$ & $>30$ \\
\hline & 2.0 & $>30$ & $>30$ & $>30$ & $>30$ & $>30$ & $>30$ & $>30$ & $>30$ & $>30$ & $>30$ & $>30$ & $>30$ & $>30$ \\
\hline
\end{tabular}

Table S4. Configurations of initial simulation systems for determining critical concentration of $\mathrm{N}_{2}$ nanobubble formation.

\begin{tabular}{|c|c|c|c|}
\hline Number & $\boldsymbol{N}_{\mathbf{2}}$ Conc.(g/L) & No. of $\boldsymbol{N}_{\mathbf{2}}$ & No. of water \\
\hline $\mathbf{1}$ & 10.0 & 85 & 3290 \\
\hline $\mathbf{2}$ & 12.0 & 101 & 3274 \\
\hline $\mathbf{3}$ & 14.0 & 117 & 3258 \\
\hline $\mathbf{4}$ & 16.0 & 133 & 3242 \\
\hline
\end{tabular}




\begin{tabular}{|c|c|c|c|}
\hline $\mathbf{5}$ & 18.0 & 149 & 3226 \\
\hline $\mathbf{6}$ & 20.0 & 165 & 3210 \\
\hline $\mathbf{7}$ & 22.0 & 181 & 3194 \\
\hline $\mathbf{8}$ & 24.0 & 196 & 3179 \\
\hline $\mathbf{9}$ & 26.0 & 211 & 3164 \\
\hline $\mathbf{1 0}$ & 28.0 & 227 & 3148 \\
\hline $\mathbf{1 1}$ & 30.0 & 242 & 3133 \\
\hline
\end{tabular}

Table S5. Benchmark setups for determining LJ parameters of G1-Na and G1-C1 non-bonded interactions.

\begin{tabular}{|c|c|c|c|c|}
\hline \multicolumn{5}{|c|}{ Group 1} \\
\hline \multicolumn{2}{|c|}{ Cross-term } & $\varepsilon(\mathrm{kJ} / \mathrm{mol})$ & Attractive term & Repulsive term \\
\hline \multicolumn{2}{|c|}{ G1-G1 $(\sigma=0.36 \mathrm{~nm})$} & 1.6 & $1.3931 \mathrm{E}-02$ & $3.0326 \mathrm{E}-05$ \\
\hline \multicolumn{2}{|c|}{ G1-P4 $(\sigma=0.42 \mathrm{~nm})$} & 1.4 & $3.0739 \mathrm{E}-02$ & $1.6873 \mathrm{E}-04$ \\
\hline \multicolumn{2}{|c|}{ G1-Q0 $(\sigma=0.49 \mathrm{~nm})$} & 1.4 & $7.7511 \mathrm{E}-02$ & $1.0729 \mathrm{E}-03$ \\
\hline \multicolumn{2}{|c|}{$\mathrm{G} 1-\mathrm{Qa}(\sigma=0.49 \mathrm{~nm})$} & 1.4 & $7.7511 \mathrm{E}-02$ & $1.0729 \mathrm{E}-03$ \\
\hline \multicolumn{2}{|c|}{$\mathrm{G} 1-\mathrm{C} 1(\sigma=0.42 \mathrm{~nm})$} & 1.6 & $3.5130 \mathrm{E}-02$ & $1.9283 \mathrm{E}-04$ \\
\hline \multirow{3}{*}{$\begin{array}{c}\mathrm{G} 1-\mathrm{Na} \\
(\sigma=0.42 \mathrm{~nm})\end{array}$} & $\mathrm{a}$ & 1.4 & $3.0739 \mathrm{E}-02$ & $1.6873 \mathrm{E}-04$ \\
\hline & $\mathrm{b}$ & 1.5 & $3.2934 \mathrm{E}-02$ & $1.8078 \mathrm{E}-04$ \\
\hline & $\mathrm{c}$ & 1.6 & $3.5130 \mathrm{E}-02$ & $1.9283 \mathrm{E}-04$ \\
\hline
\end{tabular}

\begin{tabular}{|c|c|c|c|c|}
\hline \multicolumn{5}{|c|}{ Group 2} \\
\hline \multicolumn{2}{|c|}{ Cross-term } & $\varepsilon(\mathrm{kJ} / \mathrm{mol})$ & Attractive term & Repulsive term \\
\hline \multicolumn{2}{|c|}{ G1-G1 $(\sigma=0.36 \mathrm{~nm})$} & 1.6 & $1.3931 \mathrm{E}-02$ & $3.0326 \mathrm{E}-05$ \\
\hline \multicolumn{2}{|c|}{ G1-P4 $(\sigma=0.42 \mathrm{~nm})$} & 1.4 & 3.0739E-02 & $1.6873 \mathrm{E}-04$ \\
\hline \multicolumn{2}{|c|}{ G1-Q0 $(\sigma=0.49 \mathrm{~nm})$} & 1.4 & $7.7511 \mathrm{E}-02$ & $1.0729 \mathrm{E}-03$ \\
\hline \multicolumn{2}{|c|}{ G1-Qa $(\sigma=0.49 \mathrm{~nm})$} & 1.4 & $7.7511 \mathrm{E}-02$ & $1.0729 \mathrm{E}-03$ \\
\hline \multicolumn{2}{|c|}{$\mathrm{G} 1-\mathrm{C} 1(\sigma=0.42 \mathrm{~nm})$} & 1.7 & $3.7325 \mathrm{E}-02$ & $2.0488 \mathrm{E}-04$ \\
\hline \multirow{4}{*}{$\begin{array}{c}\mathrm{G} 1-\mathrm{Na} \\
(\sigma=0.42 \mathrm{~nm})\end{array}$} & $\mathrm{a}$ & 1.4 & 3.0739E-02 & $1.6873 \mathrm{E}-04$ \\
\hline & $\mathrm{b}$ & 1.5 & $3.2934 \mathrm{E}-02$ & $1.8078 \mathrm{E}-04$ \\
\hline & $\mathrm{c}$ & 1.6 & $3.5130 \mathrm{E}-02$ & $1.9283 \mathrm{E}-04$ \\
\hline & $\mathrm{d}$ & 1.7 & $3.7325 \mathrm{E}-02$ & $2.0488 \mathrm{E}-04$ \\
\hline
\end{tabular}

\begin{tabular}{|c|c|c|c|}
\hline \multicolumn{4}{|c|}{ Group 3 } \\
\hline Cross-term & $\boldsymbol{\varepsilon}(\mathbf{k J} / \mathbf{m o l})$ & Attractive term & Repulsive term \\
\hline G1-G1 $(\sigma=0.36 \mathrm{~nm})$ & 1.6 & $1.3931 \mathrm{E}-02$ & $3.0326 \mathrm{E}-05$ \\
\hline $\mathrm{G} 1-\mathrm{P} 4(\sigma=0.42 \mathrm{~nm})$ & 1.4 & $3.0739 \mathrm{E}-02$ & $1.6873 \mathrm{E}-04$ \\
\hline $\mathrm{G} 1-\mathrm{Q} 0(\sigma=0.49 \mathrm{~nm})$ & 1.4 & $7.7511 \mathrm{E}-02$ & $1.0729 \mathrm{E}-03$ \\
\hline $\mathrm{G} 1-\mathrm{Qa}(\sigma=0.49 \mathrm{~nm})$ & 1.4 & $7.7511 \mathrm{E}-02$ & $1.0729 \mathrm{E}-03$ \\
\hline $\mathrm{G} 1-\mathrm{C} 1(\sigma=0.42 \mathrm{~nm})$ & 1.8 & $3.9521 \mathrm{E}-02$ & $2.1693 \mathrm{E}-04$ \\
\hline
\end{tabular}




\begin{tabular}{|c|c|c|c|c|}
\hline \multirow{4}{*}{$\begin{array}{c}\mathrm{G} 1-\mathrm{Na} \\
(\sigma=0.42 \mathrm{~nm})\end{array}$} & $\mathrm{a}$ & 1.4 & $3.0739 \mathrm{E}-02$ & $1.6873 \mathrm{E}-04$ \\
\cline { 2 - 5 } & $\mathrm{b}$ & 1.5 & $3.2934 \mathrm{E}-02$ & $1.8078 \mathrm{E}-04$ \\
\cline { 2 - 5 } & $\mathrm{c}$ & 1.6 & $3.5130 \mathrm{E}-02$ & $1.9283 \mathrm{E}-04$ \\
\cline { 2 - 5 } & $\mathrm{d}$ & 1.7 & $3.7325 \mathrm{E}-02$ & $2.0488 \mathrm{E}-04$ \\
\hline & $\mathrm{d}$ & 1.8 & $3.9521 \mathrm{E}-02$ & $2.1693 \mathrm{E}-04$ \\
\hline
\end{tabular}

\begin{tabular}{|c|c|c|c|c|}
\hline \multicolumn{5}{|c|}{ Group 4} \\
\hline \multicolumn{2}{|c|}{ Cross-term } & $\varepsilon(\mathrm{kJ} / \mathrm{mol})$ & Attractive term & Repulsive term \\
\hline \multicolumn{2}{|c|}{ G1-G1 $(\sigma=0.36 \mathrm{~nm})$} & 1.6 & $1.3931 \mathrm{E}-02$ & $3.0326 \mathrm{E}-05$ \\
\hline \multicolumn{2}{|c|}{$\mathrm{G} 1-\mathrm{P} 4(\sigma=0.42 \mathrm{~nm})$} & 1.4 & $3.0739 \mathrm{E}-02$ & $1.6873 \mathrm{E}-04$ \\
\hline \multicolumn{2}{|c|}{ G1-Q0 $(\sigma=0.49 \mathrm{~nm})$} & 1.4 & $7.7511 \mathrm{E}-02$ & $1.0729 \mathrm{E}-03$ \\
\hline \multicolumn{2}{|c|}{ G1-Qa $(\sigma=0.49 \mathrm{~nm})$} & 1.4 & $7.7511 \mathrm{E}-02$ & $1.0729 \mathrm{E}-03$ \\
\hline \multicolumn{2}{|c|}{$\mathrm{G} 1-\mathrm{C} 1(\sigma=0.42 \mathrm{~nm})$} & 1.9 & $4.1717 \mathrm{E}-02$ & $2.2898 \mathrm{E}-04$ \\
\hline \multirow{6}{*}{$\begin{array}{c}\mathrm{G} 1-\mathrm{Na} \\
(\sigma=0.42 \mathrm{~nm})\end{array}$} & $\mathrm{a}$ & 1.4 & $3.0739 \mathrm{E}-02$ & $1.6873 \mathrm{E}-04$ \\
\hline & $\mathrm{b}$ & 1.5 & $3.2934 \mathrm{E}-02$ & $1.8078 \mathrm{E}-04$ \\
\hline & $\mathrm{c}$ & 1.6 & $3.5130 \mathrm{E}-02$ & $1.9283 \mathrm{E}-04$ \\
\hline & d & 1.7 & $3.7325 \mathrm{E}-02$ & $2.0488 \mathrm{E}-04$ \\
\hline & $\mathrm{e}$ & 1.8 & $3.9521 \mathrm{E}-02$ & $2.1693 \mathrm{E}-04$ \\
\hline & $\mathrm{f}$ & 1.9 & $4.1717 \mathrm{E}-02$ & $2.2898 \mathrm{E}-04$ \\
\hline
\end{tabular}

\begin{tabular}{|c|c|c|c|c|}
\hline \multicolumn{5}{|c|}{ Group 5} \\
\hline \multicolumn{2}{|c|}{ Cross-term } & $\varepsilon(\mathrm{kJ} / \mathrm{mol})$ & Attractive term & Repulsive term \\
\hline \multicolumn{2}{|c|}{ G1-G1 $(\sigma=0.36 \mathrm{~nm})$} & 1.6 & $1.3931 \mathrm{E}-02$ & $3.0326 \mathrm{E}-05$ \\
\hline \multicolumn{2}{|c|}{$\mathrm{G} 1-\mathrm{P} 4(\sigma=0.42 \mathrm{~nm})$} & 1.4 & 3.0739E-02 & $1.6873 \mathrm{E}-04$ \\
\hline \multicolumn{2}{|c|}{$\mathrm{G} 1-\mathrm{Q} 0(\sigma=0.49 \mathrm{~nm})$} & 1.4 & $7.7511 \mathrm{E}-02$ & $1.0729 \mathrm{E}-03$ \\
\hline \multicolumn{2}{|c|}{$\mathrm{G} 1-\mathrm{Qa}(\sigma=0.49 \mathrm{~nm})$} & 1.4 & $7.7511 \mathrm{E}-02$ & $1.0729 \mathrm{E}-03$ \\
\hline \multicolumn{2}{|c|}{$\mathrm{G} 1-\mathrm{C} 1(\sigma=0.42 \mathrm{~nm})$} & 2.0 & $4.3912 \mathrm{E}-02$ & $2.4104 \mathrm{E}-04$ \\
\hline \multirow{7}{*}{$\begin{array}{c}\mathrm{G} 1-\mathrm{Na} \\
(\sigma=0.42 \mathrm{~nm})\end{array}$} & $\mathrm{a}$ & 1.4 & 3.0739E-02 & $1.6873 \mathrm{E}-04$ \\
\hline & $\mathrm{b}$ & 1.5 & 3.2934E-02 & $1.8078 \mathrm{E}-04$ \\
\hline & $\mathrm{c}$ & 1.6 & $3.5130 \mathrm{E}-02$ & $1.9283 \mathrm{E}-04$ \\
\hline & d & 1.7 & $3.7325 \mathrm{E}-02$ & $2.0488 \mathrm{E}-04$ \\
\hline & e & 1.8 & 3.9521E-02 & $2.1693 \mathrm{E}-04$ \\
\hline & $\mathrm{f}$ & 1.9 & 4.1717E-02 & $2.2898 \mathrm{E}-04$ \\
\hline & $\mathrm{g}$ & 2.0 & 4.3912E-02 & $2.4104 \mathrm{E}-04$ \\
\hline
\end{tabular}

\begin{tabular}{|c|c|c|c|}
\hline \multicolumn{4}{|c|}{ Group 6 } \\
\hline Cross-term & $\boldsymbol{\varepsilon}(\mathbf{k J} / \mathbf{m o l})$ & Attractive term & Repulsive term \\
\hline G1-G1 $(\sigma=0.36 \mathrm{~nm})$ & 1.6 & $1.3931 \mathrm{E}-02$ & $3.0326 \mathrm{E}-05$ \\
\hline
\end{tabular}




\begin{tabular}{|c|c|c|c|c|}
\hline \multicolumn{2}{|c|}{$\mathrm{G} 1-\mathrm{P} 4(\sigma=0.42 \mathrm{~nm})$} & 1.4 & 3.0739E-02 & $1.6873 \mathrm{E}-04$ \\
\hline \multicolumn{2}{|c|}{ G1-Q0 $(\sigma=0.49 \mathrm{~nm})$} & 1.4 & $7.7511 \mathrm{E}-02$ & $1.0729 \mathrm{E}-03$ \\
\hline \multicolumn{2}{|c|}{$\mathrm{G} 1-\mathrm{Qa}(\sigma=0.49 \mathrm{~nm})$} & 1.4 & $7.7511 \mathrm{E}-02$ & $1.0729 \mathrm{E}-03$ \\
\hline \multicolumn{2}{|c|}{$\mathrm{G} 1-\mathrm{C} 1(\sigma=0.42 \mathrm{~nm})$} & 2.1 & $4.6108 \mathrm{E}-02$ & $2.5309 \mathrm{E}-04$ \\
\hline \multirow{8}{*}{$\begin{array}{c}\mathrm{G} 1-\mathrm{Na} \\
(\sigma=0.42 \mathrm{~nm})\end{array}$} & $\mathrm{a}$ & 1.4 & 3.0739E-02 & $1.6873 \mathrm{E}-04$ \\
\hline & $\mathrm{b}$ & 1.5 & 3.2934E-02 & $1.8078 \mathrm{E}-04$ \\
\hline & $\mathrm{c}$ & 1.6 & $3.5130 \mathrm{E}-02$ & $1.9283 \mathrm{E}-04$ \\
\hline & d & 1.7 & $3.7325 \mathrm{E}-02$ & $2.0488 \mathrm{E}-04$ \\
\hline & $\mathrm{e}$ & 1.8 & $3.9521 \mathrm{E}-02$ & $2.1693 \mathrm{E}-04$ \\
\hline & $\mathrm{f}$ & 1.9 & 4.1717E-02 & $2.2898 \mathrm{E}-04$ \\
\hline & $\mathrm{g}$ & 2.0 & 4.3912E-02 & $2.4104 \mathrm{E}-04$ \\
\hline & $\mathrm{h}$ & 2.1 & $4.6108 \mathrm{E}-02$ & $2.5309 \mathrm{E}-04$ \\
\hline
\end{tabular}

\begin{tabular}{|c|c|c|c|c|}
\hline \multicolumn{5}{|c|}{ Group 7} \\
\hline \multicolumn{2}{|c|}{ Cross-term } & $\varepsilon(\mathrm{kJ} / \mathrm{mol})$ & Attractive term & Repulsive term \\
\hline \multicolumn{2}{|c|}{ G1-G1 $(\sigma=0.36 \mathrm{~nm})$} & 1.6 & $1.3931 \mathrm{E}-02$ & $3.0326 \mathrm{E}-05$ \\
\hline \multicolumn{2}{|c|}{$\mathrm{G} 1-\mathrm{P} 4(\sigma=0.42 \mathrm{~nm})$} & 1.4 & $3.0739 \mathrm{E}-02$ & $1.6873 \mathrm{E}-04$ \\
\hline \multicolumn{2}{|c|}{ G1-Q0 $(\sigma=0.49 \mathrm{~nm})$} & 1.4 & $7.7511 \mathrm{E}-02$ & $1.0729 \mathrm{E}-03$ \\
\hline \multicolumn{2}{|c|}{ G1-Qa $(\sigma=0.49 \mathrm{~nm})$} & 1.4 & $7.7511 \mathrm{E}-02$ & $1.0729 \mathrm{E}-03$ \\
\hline \multicolumn{2}{|c|}{$\mathrm{G} 1-\mathrm{C} 1(\sigma=0.42 \mathrm{~nm})$} & 2.2 & $4.8303 \mathrm{E}-02$ & $2.6514 \mathrm{E}-04$ \\
\hline \multirow{9}{*}{$\begin{array}{c}\mathrm{G} 1-\mathrm{Na} \\
(\sigma=0.42 \mathrm{~nm})\end{array}$} & $\mathrm{a}$ & 1.4 & $3.0739 \mathrm{E}-02$ & $1.6873 \mathrm{E}-04$ \\
\hline & $\mathrm{b}$ & 1.5 & $3.2934 \mathrm{E}-02$ & $1.8078 \mathrm{E}-04$ \\
\hline & $\mathrm{c}$ & 1.6 & $3.5130 \mathrm{E}-02$ & $1.9283 \mathrm{E}-04$ \\
\hline & $\mathrm{d}$ & 1.7 & $3.7325 \mathrm{E}-02$ & $2.0488 \mathrm{E}-04$ \\
\hline & $\mathrm{e}$ & 1.8 & $3.9521 \mathrm{E}-02$ & $2.1693 \mathrm{E}-04$ \\
\hline & $\mathrm{f}$ & 1.9 & $4.1717 \mathrm{E}-02$ & $2.2898 \mathrm{E}-04$ \\
\hline & $\mathrm{g}$ & 2.0 & $4.3912 \mathrm{E}-02$ & $2.4104 \mathrm{E}-04$ \\
\hline & $\mathrm{h}$ & 2.1 & $4.6108 \mathrm{E}-02$ & $2.5309 \mathrm{E}-04$ \\
\hline & $\mathrm{i}$ & 2.2 & $4.8303 \mathrm{E}-02$ & $2.6514 \mathrm{E}-04$ \\
\hline
\end{tabular}


Group 1

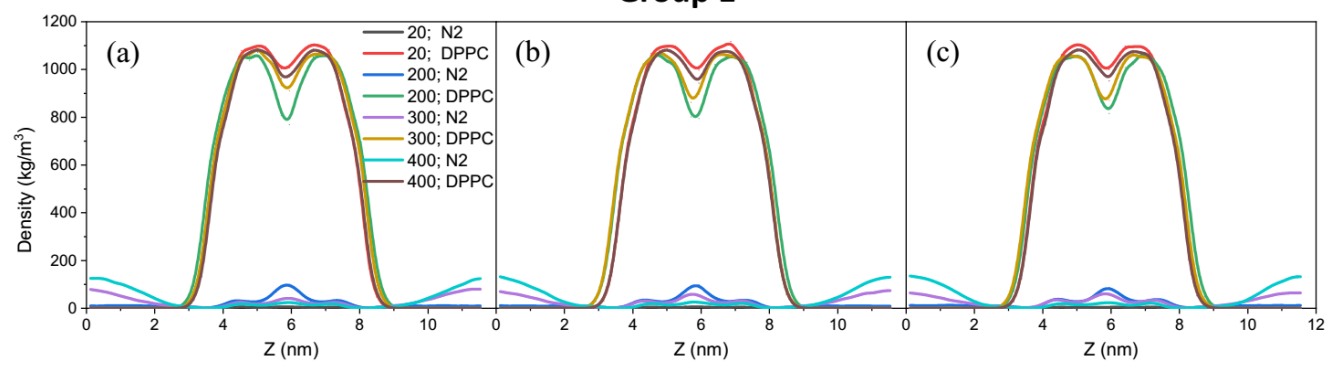

Group 2

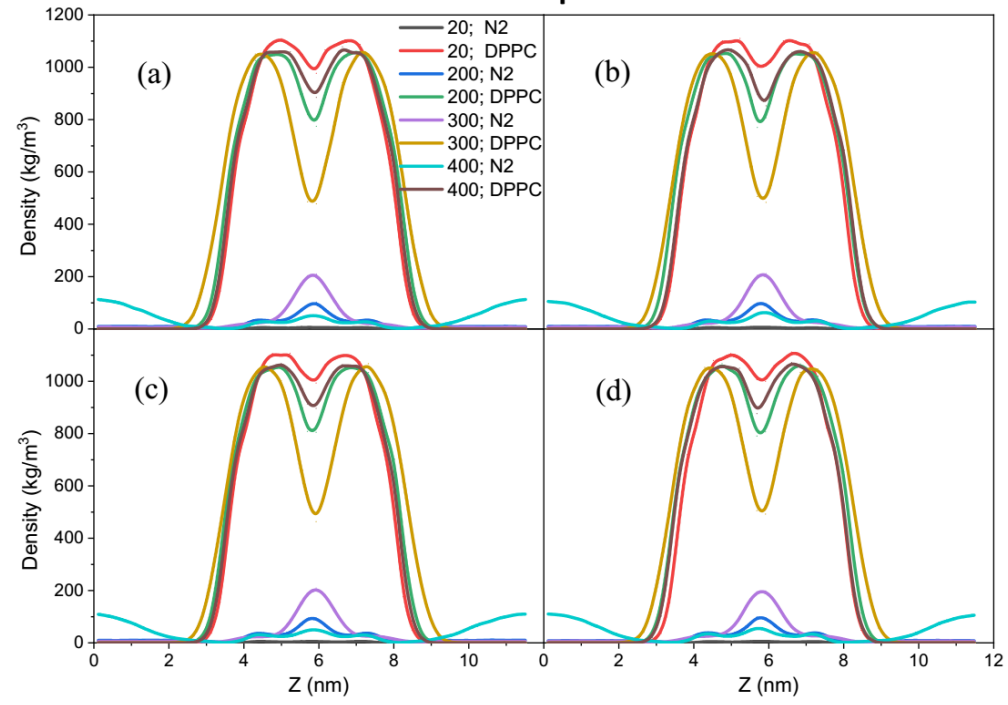

Group 3

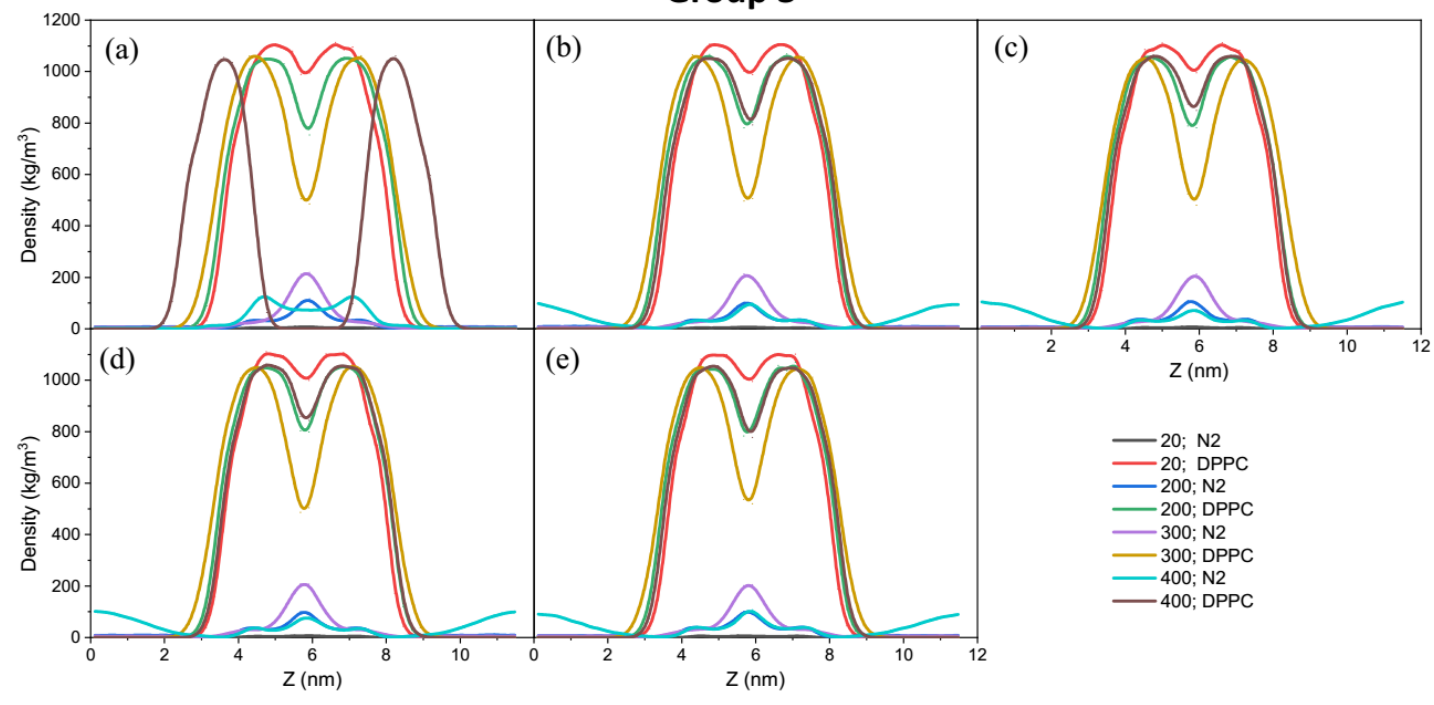




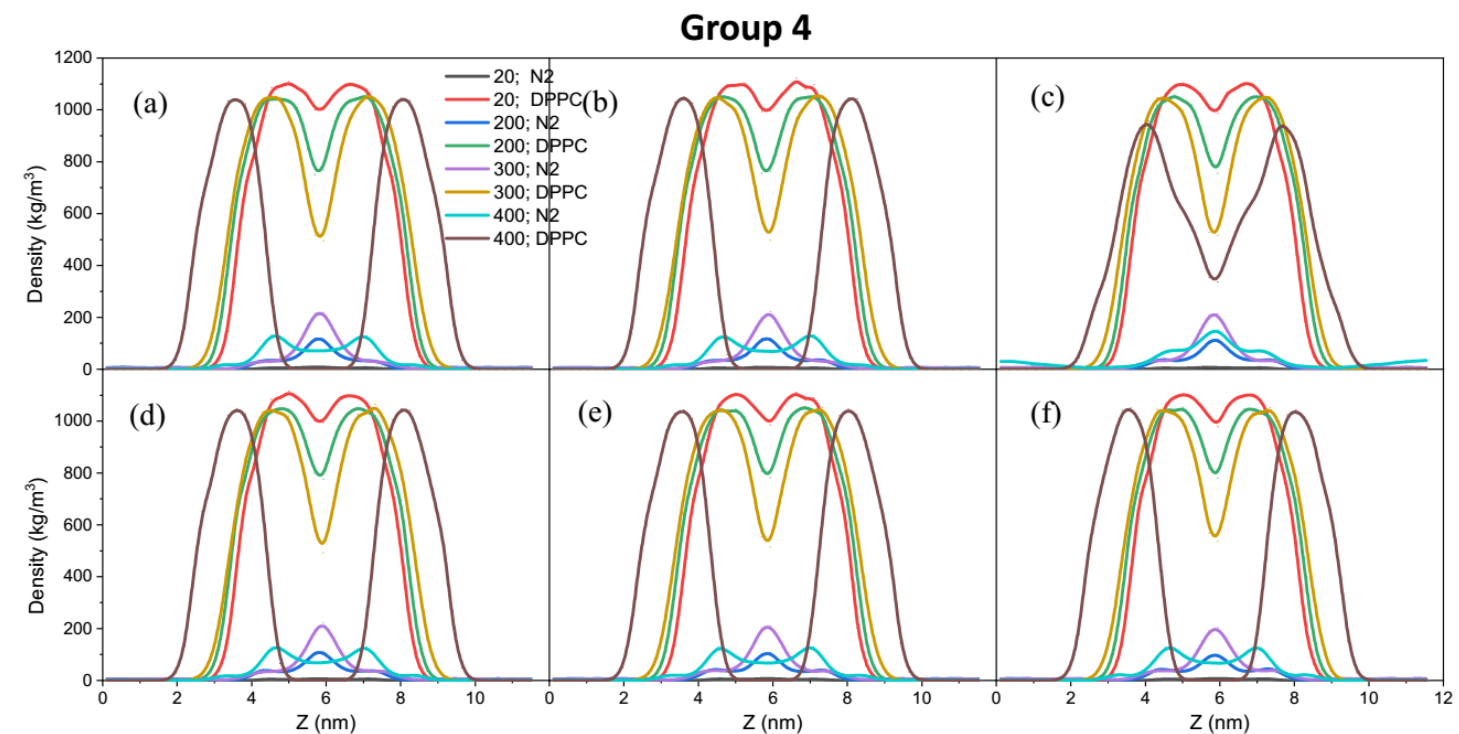

Group 5

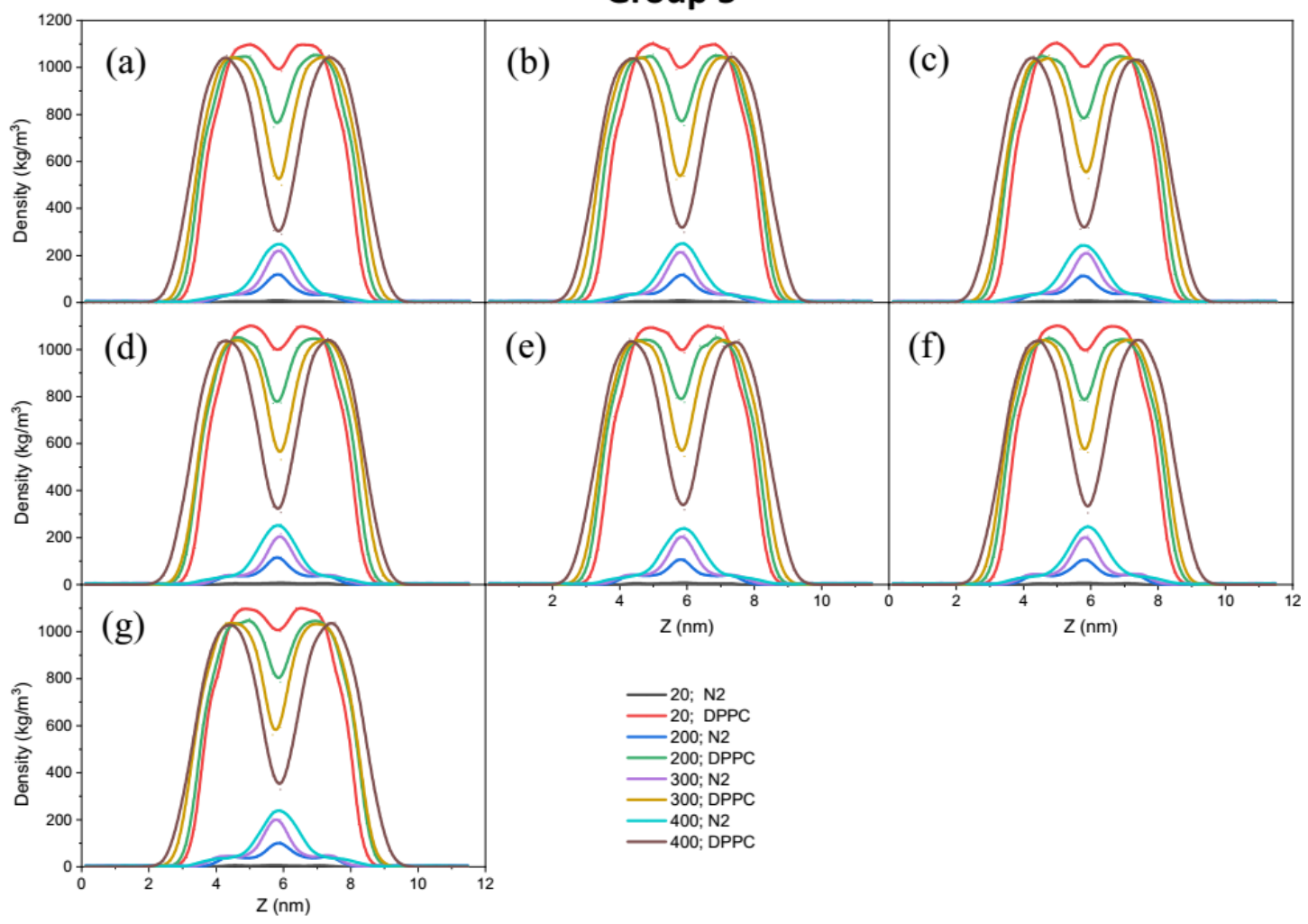



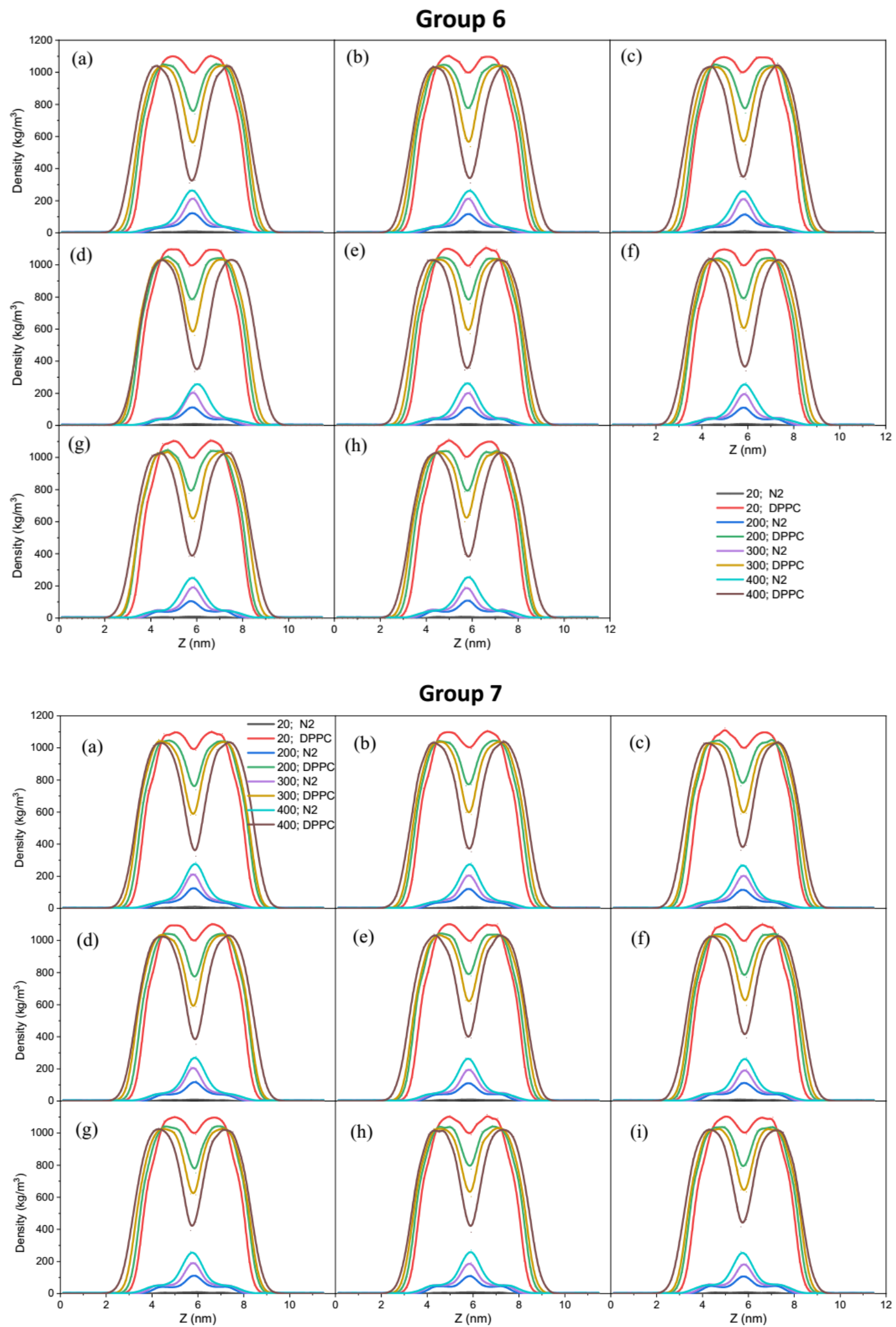
Figure S2. Density profiles of $N_{2}$ gas and DPPC molecules along the membrane normal based on G1-C1 and G1-Na interaction groups 1-7 of Table S5. For each LJ parameter set, four 128-DPPC bilayer systems with 20,200,300 or $400 N_{2}$ gas molecules initially were evenly dispersed in bulk water region and their behaviors were investigated.

Table S6. Lipid nanobubble self-assembly simulations with different initial nitrogen nanobubble radius and different number of DPPC molecules.

\begin{tabular}{|c|c|c|c|c|c|c|}
\hline System & DPPC & $\mathbf{N}_{\mathbf{2}}$ & Radius (nm) & $\mathbf{N}_{\mathbf{2}}$ nanobubble $\boldsymbol{\rho}(\mathbf{g} / \mathbf{L})$ & $\mathbf{H}_{\mathbf{2}} \mathbf{O}$ & Results \\
\hline 1 & 180 & 454 & 3 & 186.74 & 12844 & Lipid nanobubble \\
\hline 2 & 180 & 606 & 3 & 249.26 & 12844 & Lipid nanobubble \\
\hline 3 & 180 & 734 & 3.5 & 190.12 & 12284 & Lipid nanobubble \\
\hline 4 & 180 & 979 & 3.5 & 253.59 & 12284 & Lipid nanobubble \\
\hline 5 & 180 & 1084 & 4 & 188.10 & 11584 & Lipid nanobubble \\
\hline 6 & 180 & 1446 & 4 & 250.92 & 11584 & Semi-lipid nanobubble \\
\hline 7 & 256 & 438 & 3 & 180.16 & 13072 & Tubular shape \\
\hline 8 & 256 & 876 & 3 & 360.32 & 13072 & Lipid nanobubble \\
\hline 9 & 256 & 1091 & 4 & 189.32 & 11766 & Lipid nanobubble \\
\hline 10 & 256 & 2182 & 4 & 378.63 & 11766 & Tubular shape \\
\hline 11 & 256 & 1400 & 5 & 124.38 & 9749 & Lipid nanobubble \\
\hline 12 & 256 & 2100 & 5 & 186.58 & 9749 & Tubular shape \\
\hline
\end{tabular}

Table S7. Simulation systems of three-component lipid nanobubble.

\begin{tabular}{|c|c|c|c|c|c|c|}
\hline System & DPPC & DUPC & CHOL & $\mathbf{N}_{\mathbf{2}}$ & $\mathbf{H}_{\mathbf{2}} \mathbf{O}$ & $\mathbf{N}_{\mathbf{2}}$ Conc.(g/L) \\
\hline N1 & 117 & 70 & 48 & 646 & 8322 & 30.19 \\
\hline N2 & 209 & 125 & 84 & 1645 & 19512 & 32.79 \\
\hline N3 & 327 & 196 & 131 & 2435 & 32027 & 29.57 \\
\hline N4 & 471 & 282 & 189 & 3431 & 44445 & 30.02 \\
\hline N5 & 641 & 384 & 257 & 4653 & 59961 & 30.18 \\
\hline N6 & 837 & 502 & 336 & 6130 & 78938 & 30.20 \\
\hline N7 & 1060 & 636 & 424 & 7500 & 99609 & 29.28 \\
\hline N8 & 1308 & 785 & 524 & 9000 & 120367 & 29.08 \\
\hline
\end{tabular}

Table S8. Simulation system of three-component lipid bi-monolayer.

\begin{tabular}{|c|c|c|c|c|c|c|}
\hline \multirow{2}{*}{ System } & \multirow{2}{*}{$\mathbf{A}\left(\mathbf{n m}^{\mathbf{2}}\right)$} & \multicolumn{3}{|c|}{ One leaflet } & \multirow{2}{*}{$\mathbf{N}_{\mathbf{2}}$} & \multirow{2}{*}{$\mathbf{H}_{\mathbf{2}} \mathbf{O}$} \\
\cline { 3 - 5 } & & DPPC & DUPC & $\mathbf{C H O L}$ & & \\
\hline M8 & 0.64 & 1308 & 785 & 524 & 485 & 94136 \\
\hline
\end{tabular}


Table S9. Simulation system of three-component lipid bilayer.

\begin{tabular}{|c|c|c|c|c|c|c|c|}
\hline \multirow{2}{*}{ System } & \multicolumn{3}{|c|}{ Outer leaflet } & \multicolumn{3}{c|}{ Inner leaflet } & \multirow{2}{*}{$\mathrm{H}_{2} \mathbf{O}$} \\
\cline { 2 - 7 } & DPPC & DUPC & CHOL & DPPC & DUPC & CHOL & \\
\hline B8 & 1308 & 785 & 524 & 1308 & 785 & 524 & 91264 \\
\hline
\end{tabular}

\section{References:}

[1] Marrink, S. J.; Risselada, H. J.; Yefimov, S.; Tieleman, D. P.; de Vries, A. H. The Martini Force Field: Coarse Grained Model for Biomolecular Simulations. J. Phys. Chem. B 2007, 111, 78127824. 